\title{
Pricing CoCos with a Market Trigger
}

\author{
José Manuel Corcuera and Arturo Valdivia
}

\begin{abstract}
Contingent Convertible Bonds, or CoCos, are contingent capital instruments which are converted into shares, or may suffer a principal write-down, if certain trigger event occurs. In this paper we discuss some approaches to the problem of pricing CoCos when its conversion and the other relevant credit events are triggered by the issuer's share price. We introduce a new model of partial information which aims at enhancing the market trigger approach while remaining analytically tractable. We address also CoCos having the additional feature of being callable by the issuer at a series of pre-defined dates. These callable CoCos are thus exposed to a new source of risk-referred to as extension risk-since they have no fixed maturity, and the repayment of the principal may take place at the issuer's convenience.
\end{abstract}

Keywords Contingent convertible $\cdot$ Coco bond $\cdot$ Callable bond $\cdot$ Extension risk

\section{Introduction}

The Basel Committee on banking Supervision was created in 1974, after the collapse of the German Bank Herstatt, with the aim of establishing prudential rules of trading. During the 1980s this committee was concerned with the big moral hazard of Japanese banks that distorted the competition among countries. In 1988 it formulated a set of rules, so called Basel I, to stabilize the international banking system. Basically the main rule was that each bank should hold a minimum of $8 \%$ of its total assets, where, for the valuation of the assets it was used some weights reflecting the credit risk of each asset. These measures produced a credit crunch and some criticism appeared, mainly related with the weights used to measure the risk of the different assets.

\section{J.M. Corcuera $(\varangle) \cdot$ A. Valdivia}

Faculty of Mathematics, University of Barcelona, Gran Via de Les Corts Catalanes, 585, 08007 Barcelona, Spain e-mail: jmcorcuera@ub.edu

A. Valdivia

e-mail: arturo.valdivia@ub.edu 
These weights only took into account the kind of institution borrowing or issuing the security and not what the spreads observed in the market. To amend the first Basel accords and take into account the market risk and interest-rate risk, it was started a process that concluded in 2004 with new rules, Basel II. These agreements are more complex and consider not only new rules for capitalization, with the introduction of $\mathrm{VaR}$ methodology, but also supervision and transparency rules. The regulator calculated the weights on the basis of the formula

$$
K=L G D \times \Phi\left[\frac{\Phi^{-1}(P D)}{\sqrt{1-R}}+\sqrt{\frac{R}{1-R}} \times \Phi^{-1}(0.999)\right]-P D \times L G D
$$

where $\Phi$ is the CDF of the standard normal distribution, $L G D$ is the loss in case of default, $P D$ is the probability of default, and $R$ is the correlation between the portfolio of loans and a macroeconomic risk factor, see [24] for an explanation of its underlying model. To determine the different parameters, banks were allowed to use their own models.

In 2007 a financial crisis, originated in the U.S. home loans market, quickly spread to other markets, sectors and countries, forcing the Federal Reserve and the European Central Bank to intervene in response to the collapse of the interbank market. This gave rise, in 2010, to new regulation rules, known as Basel III, that would change the financial landscape. Some securities were not going to be allowed anymore as regulatory capital and supervisors put emphasis in the fact that capital regulatory should have a real loss absorbing capacity. This is when Contingent Convertibles (CoCos) started to play an important role.

In 2002 Flannery proposed and early form of CoCo that he called Reverse Convertible Debentures, see [22]. The idea was that whenever the bank issuing such debentures reaches a market-based capital ratio that is below a pre-specified level, a sufficient number of said debentures would convert into shares at the current market price. Later, in [23], he updated the proposal and named these assets as Contingent Capital Certificates. The idea behind was in agreement with what [19] wrote in The Prudential Regulation of Banks. In this work they formulated the representation hypothesis. According to this hypothesis prudential regulation should aim at replicating the corporate governance of non-financial firms, that is, acting as a representative of the debtholders of bank, regulation should play the role of creditors in nonfinancial institutions.

A Contingent Convertible is a bond issued by a financial institution where, upon the appearance of a trigger event, related with a distress of the institution, either an automatic conversion into a predetermined number of shares takes place or a partial write-down of the bond's face value is applied. It is intended to be a loss absorbing security in the sense that in case of liquidity difficulties it produces a recapitalization of the entity.

Basel III, among other regulating measures, proposed the inclusion of CoCos as part of Additional Tier 1 Capital, where Tier 1 is, roughly speaking, the capital or the assets that the entity have, for sure, in case of crisis, and consists of Common Equity 
Tier 1 and Additional Tier 1. Chan and van Wijnbergen [5] affirm that the inclusion of Cocos in Tier 1 is a likely factor in the increase of CoCo issuances. In December 2013, the CoCo market had reached $\$ 49$ bn in size in Europe.

It is a controversial issue if $\operatorname{CoCos}$ are a stabilizing security. Koziol and Lawrenz [28] show that, under certain modelling assumptions, if CoCos are part of the capital structure of the company equity holders can take more risky strategies, trying to maximize the value of their shares. In their work Koziol and Lawrenz use a low level of the asset price of the company as a trigger for the conversion. Chan and van Wijnbergen [5] point out that conversion can be seen as a negative signal by the depositors of a bank and to produce bank runs. They also argue that far from lowering the risks, CoCos can increase even the systemic risks. On the other hand [20] defend that CoCos is an appropriate solution that does not lead to moral hazard provided that conversion is tied to exogenous macroeconomic shocks.

There is also disagreement about how to establish the trigger event. It is perhaps the most controversial parameter in a CoCo. Some advocate conversion based on book values, like the different capital ratios used in Basel III. Others defend market triggers like the market value of the equity. So far the CoCos issued by the private sector are based on accounting ratios.

The market for contingent convertibles started in December 2009 when the Lloyds Banking Group launched its \$13.7 bn issue of Enhanced Capital Notes. Next in line was Rabobank making its first entry in the market for contingent debt with a $€ 1.25$ bn issue early 2010. After this, things turned quiet until February 2011, when Credit Suisse launched its so-called Buffer Capital Notes (\$2 bn). This Credit Suisse issue was done on the back of the new regulatory regime in Switzerland. This was called the "Swiss Finish" and it required the larger banks such as UBS and Credit Suisse to hold loss absorbing capital up to $19 \%$ of their risk weighted assets, see [11]. This capital had to consist of at least $10 \%$ common equity and up to $9 \%$ in contingent capital. In 2014 a number of banks issued CoCos, including Deutsche Bank and Mizuho Financial Group.

From a modelling point of view and sometimes depending on the trigger chosen for the conversion, usually a low level of a certain index related with the asset, the debt or the equity of the firm, one can follow an intensity approach or a structural approach to model the trigger. For an intensity approach for modelling the conversion time, see for instance $[8,16]$. This approach is especially useful when pricing CoCos is the main interest, it is a kind of statistical modelling of the trigger event. In fact what one models is the law of the conversion time. In the structural approach for modelling the trigger, one models the random variable describing the conversion time and one relates it with the dynamics of the assets, debt, or equities. It is a more explanatory approach, where one can use the observed dynamics of certain economic facts to describe the conversion time.

In the structural approach one can use a market trigger based on a low level of the equity value. This approach is very appealing because the market value of equity is an observable economic variable whose dynamics can be modelled in order to fit historical data. At the same time it allows to obtain close pricing formulas, like in [13], and to define an objective trigger that can be observed immediately. Cheridito 
and $\mathrm{Xu}$ [7] also use this trigger and show that pricing and hedging problems can be treated for quite general continuous models and barriers and that solutions can always be obtained, at least numerically, using Feymann-Kac type results, translating the problem of pricing into a problem of solving a series of parabolic partial differential equations (PDE) with Dirichlet boundary conditions.

One argument against accounting triggers is that monitoring is not continuous, there is always a delay in the information. Moreover, in the recent crisis these triggers did not provide any signal of distress in troubled banks. On the contrary, when using market triggers, there exist the risk of market manipulations of the equity price trying to force the conversion or undesirable phenomena like the death-spiral effect. In [17] authors propose a system of multiple triggers to avoid the death spiral, whereas in [13] a system of coupon cancellations is proposed in order to alleviate this effect. Sundaresan and Wang [39] analyze this kind of trigger and find that to use low equity values as trigger is not innocuous. It can have destabilizing effects in the firm. Their reasoning is roughly speaking the following. Suppose that $\left(A_{t}\right)_{t \geq 0}$ represents the aggregate value of the assets of the company, $\left(D_{t}\right)_{t \geq 0}$ its aggregate debt, $\left(C_{t}\right)_{t \geq 0}$ the aggregate value of the $\operatorname{CoCos}$, and $\left(L_{t}\right)_{t \geq 0}$ the aggregate liquidation value of the CoCos issued by the firm. Set $\tau$ for the conversion time, and assume that it happens when the (aggregate) equity value, $\left(E_{t}\right)_{t \geq 0}$ is lower that some level say $\left(H_{t}\right)_{t \geq 0}$. Since equity value is the residual value of the asset, at any time $t \leq \tau$, we will have two possibilities:

$$
A_{t}-D_{t}-C_{t}>H_{t} \text { if } t<\tau
$$

or

$$
A_{t}-D_{t}-L_{t}<H_{t} \text { if } t=\tau \text {. }
$$

This gives that, at any time $t \leq \tau$,

$$
D_{t}+C_{t}<A_{t}<D_{t}+L_{t} .
$$

So, if $C_{t}>L_{t}$ there is not any possible value for $A_{t}$ and if $C_{t}<L_{t}$ there are multiple values, all of these allowing, according to [39], potential price manipulation, market uncertainty, inefficient allocation and unreliability of conversion.Obviously if $C_{t}=L_{t}$, that is, if there is no jump on the wealth of CoCo's investors at the conversion time, then the equilibrium is possible, but this is considered non realistic and even problematic, since a punitive conversion for shareholders could help to maintain the market discipline. As a possible remedy to this situation [32] propose a trigger based not only in the value of the equities but also in the value of the CoCos. They also propose to include, in the CoCo contract, a PUT option of the issuer on the equities, in case of conversion, to avoid market price manipulations.

Another possibility, in the structural approach, is to use a low value of the asset value as a trigger. It is also quite appealing, since it allows to consider the whole capital structure of the company, to study the effect of CoCo debt in the equity value and to obtain the optimal conversion barrier for the shareholders. See for instance 
$[6,28]$ or [2]. In this latter paper they also consider other triggers aiming at providing a proxy for regulatory triggers.

Nevertheless in all these papers authors consider a fixed maturity of the CoCo bond. However bonds often do not just have a legal maturity but can have also different call dates. In such cases, the bond can be called back by the issuer at these dates prior to the legal maturity. This risk of extending the life of a contract is what we call extension risk. This has been treated for the first time in [18] using an intensity approach and in [14] using a structural approach.

In this paper we review our work on the topic of pricing $\mathrm{CoCos}$, and we introduce new issues like delay in the information and jumps. We always consider low values of the stock as triggers and $\mathrm{CoCos}$ that convert totally into equities in case of conversion. The paper is organized as follows. The contract features are specified in Sect. 2. In addition, a model-free formula for the CoCoprice is presented in order to establish the general pricing problem. In Sect. 3 a model with stochastic interest rates is studied. A closed-form formula for the price is given and subsequently used in order to study the Black-Scholes model and its Greeks. In Sect. 4 two advanced models are discussed. On the one hand, the stochastic volatility Heston model is incorporated to the share price dynamics, and the correspondent prices are later on obtained by a PDE approach. On the other hand, an exponential Lévy model is proposed, and the obtainment of the correspondent prices is addressed by a Fourier method exploiting the so-called Wiener-Hopf factorization for Lévy processes. In Sect. 5 we introduce a new trigger model which aims to describe the delay of information present in accounting triggers. Finally, in Sect. 6 we show how the original pricing problem is modified when no fixed maturity is imposed on the CoCo. This variation leads to what we call CoCos with extension risk, and the pricing problem includes solving an optimal stopping time problem which, even in the Black-Scholes, turns out to be far from straightforward.

\section{The Pricing Problem}

The definition of a CoCo requires the specification of its face value $K$ and maturity $T$, along with the random time $\tau$ at which the CoCo conversion may occur, and the prefixed price $C_{p}$ at which the investor may buy the shares if conversion takes place. We refer to $\tau$ and $C_{p}$ as conversion time and conversion price, respectively. The quantity $C_{r}:=K / C_{p}$ is refered to as conversion ratio. Assuming $m$ coupons are attached to the $\mathrm{CoCo}$, then we further need to specify a series of credit events that may trigger a coupon cancellation. Denote by $\tau_{1}, \ldots, \tau_{m}$ the random times at which the aforementioned credit events may occur. Then the whole coupon structure $\left(c_{j}, T_{j}, \tau_{j}\right)_{j=1}^{m}$ of the CoCo is defined, in such a way that the amount $c_{j}$ is paid at time $T_{j}$, provided the $\tau_{j}>T_{j}$. We establish that the last coupon is paid at maturity time, i.e., $T_{m}:=T$. The coupon cancellation feature was introduced in [13] in order to alleviate the so-called death-spiral effect exhibited by the traditional $\mathrm{CoCo}$-see details in Sect. 3.1. Thus it is assumed that coupon cancellation precedes conversion 
according to $\tau_{1} \geq \cdots \geq \tau_{m} \geq \tau$. Of course it suffices to set $\tau_{1}=\cdots=\tau_{m}=\infty$ if this feature were to be excluded from modelling.

It will be assumed that the issuer of the CoCo pays dividends according to a deterministic function $\kappa$. From the investors side, it seems to be reasonable to assume that no dividends are paid after the conversion time $\tau$. Thus hereafter we shall assume that the following condition holds true.

Condition (F). There are no dividends after the conversion time $\tau$.

Remark 1 It is worth mentioning that, whereas this condition simplifies the expressions obtained for the price, it is not crucial, in the sense that the computations still can be carried on. We refer to [13] for a further discussion on this topic.

Once these features are settled, the CoCo's final payoff, is given by

$$
K \mathbf{1}_{\{\tau>T\}}+\frac{K}{C_{p}} S_{\tau} \mathbf{1}_{\{\tau \leq T\}}+\sum_{j=1}^{m} c_{j} \mathrm{e}^{\int_{T_{j}}^{T} r_{u} \mathrm{~d} u} \mathbf{1}_{\left\{\tau_{j}>T_{j}\right\}}
$$

where $\left(S_{t}\right)_{t \geq 0}$ and $\left(r_{t}\right)_{t \geq 0}$ stand for the share price and interest rate, respectively.

\subsection{A Model-Free Formula for the CoCo Price}

In Proposition 2, a model-free formula for the CoCo price is given. Let us first introduce some notation required for what follows. Underlying to our market, we shall consider a complete probability space $(\Omega, \mathscr{F}, \mathbb{P})$, endowed with a filtration $\mathbb{F}:=\left(\mathscr{F}_{t}\right)_{t \in[0, T]}$ representing the trader's information-this includes the information generated by all state variables (e.g., share price, interest rates, total assets value,...) and the default-free market. All filtrations considered are assumed to satisfy the usual conditions of $\mathbb{P}$-completeness and right-continuity. We shall denote the evolution of the money in the bank account by $\left(B_{t}\right)_{t \in[0, T]}$, i.e.,

$$
B_{t}=\exp \left\{\int_{0}^{t} r_{u} \mathrm{~d} u\right\}, \quad 0 \leq t \leq T .
$$

Recall that two probability measures on $(\Omega, \mathscr{F}), \mathbb{P}_{1}$ and $\mathbb{P}_{2}$, are said to be equivalent if, for every $A \in \mathscr{F}, \mathbb{P}_{1}(A)=0$ if and only if $\mathbb{P}_{2}(A)=0$. We shall assume the existence of a risk-neutral probability measure $\mathbb{P}^{*}$, equivalent to the real-world probability measure $\mathbb{P}$, such that the discounted value of self-financing portfolios, $\left(\widetilde{V}_{t}:=\frac{V_{t}}{B_{t}}\right)_{t \in[0, T]}$, follows a $\mathbb{P}^{*}$-martingale. Hereafter the symbol tilde will be used to denote discounted prices. Now, in addition to $\mathbb{P}^{*}$, we shall consider other two probability measures (also equivalent to $\mathbb{P}$ ) which will allow us to carry on some of the computations related with the CoCo arbitrage-free price. First, letting $\left(B\left(t, T_{j}\right)\right)_{t \geq 0}$ stand for the price of the default-free zero-coupon bond with maturity $T_{j}$, we define 
the $T_{j}$-forward measure $\mathbb{P}^{T_{j}}$ through its Radon-Nikodým derivative with respect to $\mathbb{P}^{*}$ as given by

$$
\frac{\mathrm{d} \mathbb{P}^{T_{j}}}{\mathrm{~d} \mathbb{P}^{*}}=\frac{\mathrm{e}^{-\int_{0}^{T_{j}} r_{u} \mathrm{~d} u}}{B\left(0, T_{j}\right)}
$$

We say that $\mathbb{P}^{T_{j}}$ is given by taking the bond price $\left(B\left(t, T_{j}\right)\right)_{t \geq 0}$ as numéraire. Similarly, but now taking the issuer's share price $\left(S_{t}\right)_{t \geq 0}$ - without dividends-as numéraire, we obtain the share measure $\mathbb{P}^{(S)}$; its Radon-Nikodým derivative with respect to $\mathbb{P}^{*}$ is given by

$$
\frac{\mathrm{d} \mathbb{P}^{(S)}}{\mathrm{d} \mathbb{P}^{*}}=\frac{\mathrm{e}^{-\int_{0}^{T}\left[r_{u}-\kappa(u)\right] \mathrm{d} u} S_{T}}{S_{0}} .
$$

In what follows, expectation with respect to $\mathbb{P}^{*}, \mathbb{P}^{T_{j}}$ and $\mathbb{P}^{(S)}$ will be denoted by $\mathbb{E}^{*}$, $\mathbb{E}^{T_{j}}$ and $\mathbb{E}^{(S)}$, respectively.

Proposition 2 The discounted CoCo arbitrage-free price, on the set $\{t<\tau\}$, equals

$$
\begin{aligned}
\widetilde{\pi}_{t} & :=\mathbb{E}^{*}\left[\widetilde{K} \mathbf{1}_{\{\tau>T\}} \mid \mathscr{F}_{t}\right]+C_{r} \mathbb{E}^{*}\left[\widetilde{S}_{T} \mathbf{1}_{\{\tau \leq T\}} \mid \mathscr{F}_{t}\right]+\sum_{j: T_{j}>t}^{m} \mathbb{E}^{*}\left[\widetilde{c}_{j} \mathbf{1}_{\left\{\tau_{j}>T_{j}\right\}} \mid \mathscr{F}_{t}\right] \\
& =K \widetilde{B}(t, T) \mathbb{P}^{T}\left(\tau>T \mid \mathscr{F}_{t}\right)+\frac{C_{r} \widetilde{S}_{t}}{\mathrm{e}^{\int_{t}^{T} K(u) \mathrm{d} u}} \mathbb{P}^{(S)}\left(\tau \leq T \mid \mathscr{F}_{t}\right)+\sum_{j: T_{j}>t}^{m} c_{j} \widetilde{B}\left(t, T_{j}\right) \mathbb{P}^{T_{j}}\left(\tau_{j}>T_{j} \mid \mathscr{F}_{t}\right) .
\end{aligned}
$$

Proof Due to the Condition (F), to receive $\frac{K}{C_{p}} S_{\tau}$ at time $\tau$ is equivalent to receive $\frac{K}{C_{p}} S_{T}$ at time $T$. Therefore the payoff in (5) is equivalent to

$$
K \mathbf{1}_{\{\tau>T\}}+\frac{K}{C_{p}} S_{T} \mathbf{1}_{\{\tau \leq T\}}+\sum_{j=1}^{m} c_{j} \mathrm{e}^{\int_{T_{j}}^{T} r_{u} \mathrm{~d} u} \mathbf{1}_{\left\{\tau_{j}>T_{j}\right\}}
$$

and thus expression (8) for the price follows by preconditioning, taking into account that $C_{r}=\frac{K}{C_{p}}$. As for (9), it suffices to notice that, in light of the abstract Bayes rule, for every $X \in \mathscr{L}^{1}\left(\mathscr{F}_{T_{j}}, \mathbb{P}^{T_{j}}\right)$ we have

$$
\mathbb{E}^{T_{j}}\left[X \mid \mathscr{F}_{t}\right]=\frac{\mathbb{E}^{*}\left[X \frac{\mathrm{d} \mathbb{P}^{T_{j}}}{\mathrm{~d} \mathbb{P}^{*}} \mid \mathscr{F}_{t}\right]}{\mathbb{E}^{*}\left[\frac{\mathrm{d} \mathbb{P}^{T_{j}}}{\mathrm{~d} \mathbb{P}^{*}} \mid \mathscr{F}_{t}\right]}=\frac{\mathbb{E}^{*}\left[X \mathrm{e}^{\left.-\int_{0}^{T_{j}} r_{u} \mathrm{~d} u \mid \mathscr{F}_{t}\right]}\right.}{\mathrm{e}^{-\int_{0}^{t} r_{u} \mathrm{~d} u} B\left(t, T_{j}\right)},
$$


and thus

$$
\mathbb{E}^{*}\left[X \mathrm{e}^{-\int_{t}^{T_{j}} r_{u} \mathrm{~d} u} \mid \mathscr{F}_{t}\right]=B\left(t, T_{j}\right) \mathbb{E}^{T_{j}}\left[X \mid \mathscr{F}_{t}\right]
$$

Similarly, for every $X \in \mathscr{L}^{1}\left(\mathscr{F}_{T}, \mathbb{P}^{(S)}\right)$ we have

$$
\mathbb{E}^{*}\left[X S_{T} \mathrm{e}^{-\int_{t}^{T} r_{u} \mathrm{~d} u} \mid \mathscr{F}_{t}\right]=\mathrm{e}^{-\int_{t}^{T} \kappa(u) \mathrm{d} u} S_{t} \mathbb{E}^{(S)}\left[X \mid \mathscr{F}_{t}\right] .
$$

Combining (8) with these identities we get the expression (9).

With Proposition 2 at hand, the subsequent difference between models relies on how conversion and coupon cancellation is defined, and how the corresponding prices are evaluated. In this paper we follow a structural approach to price CoCos. That is to say, given a model for the share price $\left(S_{t}\right)_{t \geq 0}$, a series of critical time-varying barriers $\ell$ and $\ell^{j}$ are set in such a way that $\ell^{1} \geq \cdots \geq \ell^{m} \geq \ell$ and the credit events are given by

$$
\tau:=\inf \left\{t>0: S_{t} \leq \ell(t)\right\}, \quad \text { and } \quad \tau_{j}:=\inf \left\{t>0: S_{t} \leq \ell^{j}(t)\right\}, \quad j=1, \ldots, m,
$$

with the standard convention inf $\varnothing:=\infty$. Then our main concern is to derive analytically tractable formulas for (8)-(9), either in the form of closed formulas or efficient simulation methods. Later on in Sects. 5 and 6 we shall incorporate short-term uncertainty and extension risk to the pricing problem.

\subsection{Pricing CoCos with Write-Down}

In the case of CoCos with write-down, upon the appearance of the trigger event the investor does not receive a certain amount of shares. Instead, she receives only a fraction $R \in(0,1)$ of the original face value $K$, provided that the issuer has not defaulted. Let $\delta$ denote the random time at which the issuer may default. Then the payoff of this CoCo contract with write-down equals

$$
K \mathbf{1}_{\{\tau>T\}}+R K \mathbf{1}_{\{\tau \leq T\}} \mathbf{1}_{\{\delta>T\}}+\sum_{j=1}^{m} c_{j} \mathrm{e}^{\int_{T_{j}}^{T} r_{u} \mathrm{~d} u} \mathbf{1}_{\left\{\tau_{j}>T_{j}\right\}}
$$

Similarly to Proposition 2, we can give now a model-free price formula for the CoCo with write-down. For this matter, we make no further assumption beyond model consistency in the sense that $\delta$ and $\tau$ are modelled in such a way that $\delta>\tau$ so that default may only occur after conversion. 
Proposition 3 The discounted arbitrage-free price of the CoCo with write-down, on the set $\{t<\tau\}$, equals

$$
\begin{aligned}
\widetilde{\pi}_{t}^{w d}:= & (1-R) K \widetilde{B}(t, T) \mathbb{P}^{T}\left(\tau>T \mid \mathscr{F}_{t}\right)+R K \widetilde{B}(t, T) \mathbb{P}^{T}\left(\delta>T \mid \mathscr{F}_{t}\right) \\
& +\sum_{j: T_{j}>t}^{m} c_{j} \widetilde{B}\left(t, T_{j}\right) \mathbb{P}^{T_{j}}\left(\tau_{j}>T_{j} \mid \mathscr{F}_{t}\right) .
\end{aligned}
$$

Proof It suffices to notice that the payoff can be rewritten as

$$
\begin{aligned}
R \mathbf{1}_{\{\delta>s\}} \mathbf{1}_{\{\tau \leq s\}}+\mathbf{1}_{\{\tau>s\}} & =R \mathbf{1}_{\{\delta>s\}} \mathbf{1}_{\{\tau \leq s\}}+\left(R \mathbf{1}_{\{\tau>s\}}+(1-R) \mathbf{1}_{\{\tau>s\}}\right) \\
& =R \mathbf{1}_{\{\delta>s\}} \mathbf{1}_{\{\tau \leq s\}}+R \mathbf{1}_{\{\tau>s\}} \mathbf{1}_{\{\delta>s\}}+(1-R) \mathbf{1}_{\{\tau>s\}} \\
& =R \mathbf{1}_{\{\delta>s\}}\left(\mathbf{1}_{\{\tau \leq s\}}+\mathbf{1}_{\{\tau>s\}}\right)+(1-R) \mathbf{1}_{\{\tau>s\}},
\end{aligned}
$$

where for the second equivalence we have used the identity $\mathbf{1}_{\{\tau>s\}} \mathbf{1}_{\{\delta>s\}}=\mathbf{1}_{\{\tau>s\}}$, which holds due to the consitency assumption $\tau<\delta$.

By comparing (9) with (15) we can see that the techniques used to price the CoCo with conversion can be readily applied to CoCo with write-down. Across this work we focus on the former contract.

\section{A Model with Stochastic Interest Rates}

In this section we assume that the price of default-free zero-coupon bonds are stochastic. More specifically, for $j \in\{1, \ldots, m\}$, the default-free zero-coupon bond price $\left(B\left(t, T_{j}\right)\right)_{t \in\left[0, T_{j}\right]}$ is assumed to have the following $\mathbb{P}^{*}$-dynamics

$$
\frac{\mathrm{d} B\left(t, T_{j}\right)}{B\left(t, T_{j}\right)}=r_{t} \mathrm{~d} t+\sum_{k=1}^{d} b_{k}\left(t, T_{j}\right) \mathrm{d} W_{t}^{k},
$$

where each $b_{k}$ is a positive deterministic càdlàg function, and $\left(W_{t}^{1}, \ldots, W_{t}^{d}\right)_{t \in[0, T]}$ is a $d$-dimensional Brownian motion with respect to the risk-neutral probability measure $\mathbb{P}^{*}$ and the trader's filtration $\mathbb{F}$. We shall assume as well that the share price $\left(S_{t}\right)_{t \in[0, T]}$ obeys

$$
\frac{\mathrm{d} S_{t}}{S_{t}}=\left[r_{t}-\kappa(t)\right] \mathrm{d} t+\sum_{k=1}^{d} \sigma_{k}(t) \mathrm{d} W_{t}^{k}
$$

where $\sigma:=\left(\sigma_{k}\right)_{1}^{d}$ is a positive deterministic càdlàg function such that, for all $t \in$ $\left[0, T_{j}\right]$, the inequality $\left\|\sigma(t)-b\left(t, T_{j}\right)\right\|>0$ is satisfied. 
The conversion and coupon cancellation events in our model are linked to the asset dividends and the evolution of bond prices, in such a way that conversion is triggered as soon as $\left(S_{t}\right)_{t \geq 0}$ crosses

$$
\ell_{t}:=L_{m} B\left(t, T_{m}\right) \exp \left\{\int_{t}^{T_{m}} \kappa(u) \mathrm{d} u\right\}, \quad 0 \leq t \leq T_{m} ;
$$

and similarly, for $j \in\{1, \ldots, m\}$, the $j$-th coupon cancellation is triggered as soon as $\left(S_{t}\right)_{t \geq 0}$ crosses

$$
\ell_{t}^{j}:= \begin{cases}L_{j} B\left(t, T_{j}\right) \exp \left\{\int_{t}^{T_{j}} \kappa(u) \mathrm{d} u\right\}, & 0 \leq t<T_{j} \\ M_{j}, & t=T_{j} .\end{cases}
$$

The parameters $M_{j}$ and $L_{j}$ are assumed to be given non-negative constants satisfying $M_{j} \geq L_{j}$, with $L_{m}<C_{p}$ and

$$
\frac{L_{j+1}}{L_{j}} \leq \exp \left\{-\int_{T_{j}}^{T_{j+1}} \kappa(u) \mathrm{d} u\right\}, \quad j=1, \ldots, m-1,
$$

so that the required ordering $\ell_{t}^{1} \geq \ell_{t}^{2} \geq \cdots \geq \ell_{t}^{m}$ is fulfilled-thus ensuring that $0 \leq \tau_{j} \leq T_{j}$ implies $\tau_{j}<\tau_{j+1}$, for $j=1, \ldots, m-1$. Clearly the Mertonian condition (i.e., $S_{T_{j}}$ must be bigger than $M_{j}$ ) can be removed by taking $L_{j}=M_{j}$. See Fig. 1 for an illustration of the barrier's shape and parameters.

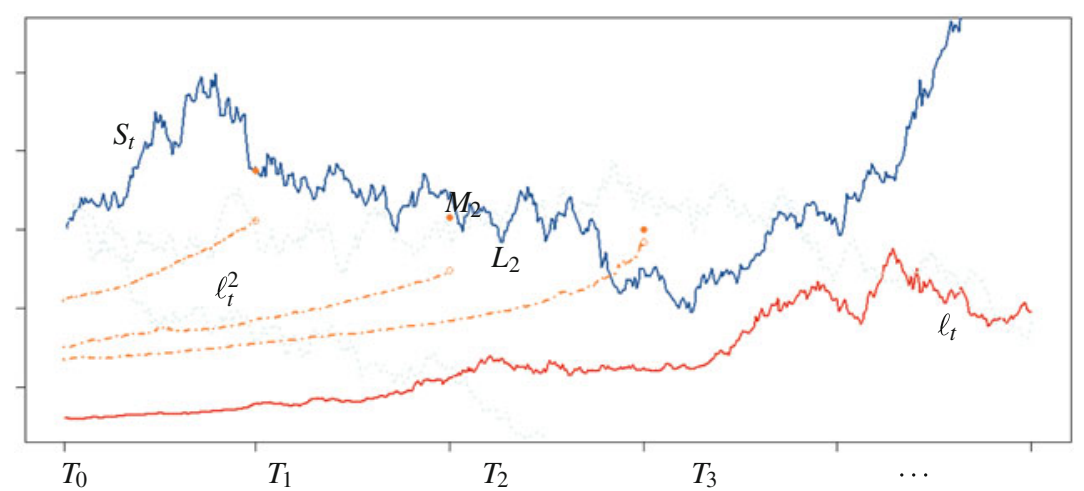

Fig. 1 The graph illustrates the share price $\left(S_{t}\right)_{t \geq 0}$, along with the barriers $\ell^{j}$ and its parameters $L_{j}$ and $M_{j}$. The first barrier is hit at $t=T_{1}$, whereas the third one is hit at some $T_{2}<t<T_{3}$. On the other hand, the second barrier is not hit since the share price stays above $\ell^{2}$ on the whole period $\left[T_{0}, T_{2}\right]$, and the Mertonian condition is satisfied, i.e., $S_{T_{2}}>M_{2}$. Conversion is not triggered either since the barrier $\ell$ is never hit by $\left(S_{t}\right)_{t \geq 0}$ 
In the current setting, the process $\left(U_{t}^{j}:=\log \frac{S_{t}}{\ell^{j}(t)}\right)_{t \geq 0}$ plays a fundamental role. Indeed, from the definition of the random times $\tau$ and $\tau_{j}$ (see (14)) and the barriers $\ell$ and $\ell^{j}$ it follows that

$$
\begin{aligned}
\left\{\tau>T_{m}\right\} & =\left\{\inf _{0 \leq t \leq T_{m}} U_{t}^{m}>0\right\} \text { and }\left\{\tau_{j}>T_{j}\right\} \\
& =\left\{\inf _{0 \leq t \leq T_{j}} U_{t}^{j}>0, U_{T_{j}}^{j}>\log \frac{M_{j}}{L_{j}}\right\}, \quad j=1, \ldots, m .
\end{aligned}
$$

From this observation we have that, in order to price the CoCo contract, we need to be able to compute the conditional joint distribution of $\left(\underline{U}_{T_{j}}^{j}, U_{T_{j}}^{j}\right)$, where we have defined $\underline{U}_{T_{j}}^{j}:=\inf _{0 \leq t \leq T_{j}} U_{t}^{j}$. To this matter, notice that an application of the Itô formula tells us that

$$
\mathrm{d} U_{t}^{j}=-\frac{1}{2}\left\|\sigma(t)-b\left(t, T_{j}\right)\right\|^{2} \mathrm{~d} t+\left\|\sigma(t)-b\left(t, T_{j}\right)\right\| \mathrm{d} W_{t}^{T_{j}} .
$$

where $\left(W_{t}^{T_{j}}\right)_{t \geq 0}$ is the $\mathbb{P}^{T_{j}}$-Brownian motion given by the Girsanov theorem, corresponding to the probability change (6). In fact we can see that under $\mathbb{P}^{(S)}$ we have similar dynamics

$$
\mathrm{d} U_{t}^{j}=\frac{1}{2}\left\|\sigma(t)-b\left(t, T_{j}\right)\right\|^{2} \mathrm{~d} t+\left\|\sigma(t)-b\left(t, T_{j}\right)\right\| \mathrm{d} W_{t}^{(S)}
$$

where $\left(W_{t}^{(S)}\right)_{t \geq 0}$ is the $\mathbb{P}^{(S)}$-Brownian motion corresponding now to the probability change (7). Consequently, a time change given by

$$
a_{j}(t):=\int_{0}^{t}\left\|\sigma(s)-b\left(s, T_{j}\right)\right\|^{2} \mathrm{~d} s, \quad 0 \leq t \leq T_{j},
$$

renders the fundamental process $\left(U_{t}^{j}:=\log \frac{S_{t}}{\ell^{j}(t)}\right)_{t \geq 0}$ a drifted Brownian motion. Then we can apply a known result (see [34]) on joint distribution of the Brownian motion and its running infimum in order to obtain the following closed-formula for the CoCo price. See details in [13].

Proposition 4 In the current setting, the CoCo arbitrage-free price, on the set $\{t<$ $\left.\tau_{m}\right\}$, is given by

$$
\begin{aligned}
\pi_{t}= & \sum_{j: T_{j}>t}^{m} \mathbf{1}_{\left\{t<\tau_{j}\right\}} c_{j}\left(B\left(t, T_{j}\right) \Phi\left(-d_{+}^{j}-D_{j}\right)-\frac{S_{t} \mathrm{e}^{-\int_{t}^{T_{j}} \kappa(u) \mathrm{d} u}}{L_{j}} \Phi\left(d_{-}^{j}-D_{j}\right)\right) \\
& +K B\left(t, T_{m}\right)+\left(C_{r}-\frac{K}{L_{m}}\right)\left(L_{m} B\left(t, T_{m}\right) \Phi\left(d_{+}^{m}\right)+S_{t} \mathrm{e}^{-\int_{t}^{T_{m}} \kappa(u) \mathrm{d} u} \Phi\left(d_{-}^{m}\right)\right),
\end{aligned}
$$


where

$$
\begin{aligned}
& d_{ \pm}^{j}=\frac{\log \frac{L_{j} B\left(t, T_{j}\right) \mathrm{e}^{\int_{t} T_{j}}}{S_{t}(u) \mathrm{d} u} \pm \frac{1}{2} \int_{t}^{T_{j}}\left\|\sigma(u)-b\left(u, T_{j}\right)\right\|^{2} \mathrm{~d} u}{\sqrt{\int_{t}^{T_{j}}\left\|\sigma(u)-b\left(u, T_{j}\right)\right\|^{2} \mathrm{~d} u}}, \quad \text { and } \\
& D_{j}=\frac{1}{\sqrt{\int_{t}^{T_{j}}\left\|\sigma(u)-b\left(u, T_{j}\right)\right\|^{2} \mathrm{~d} u}} \log \frac{M_{j}}{L_{j}}
\end{aligned}
$$

and $\Phi$ denotes the standard Gaussian cumulative distribution function.

\subsection{The Black-Scholes Model and the Greeks}

The Black-Scholes model is obtained as a particular case of the model above, by taking default-free bond prices with null volatilities-i.e., by taking the $b\left(\cdot, T_{j}\right)$ in (16) to be zero. Consequently, the closed-form price formula given by Proposition 4 can be used in order to derive the Greeks, Delta $\Delta$ and Vega v, which respectively describe the CoCo's price sensitivity to share price and volatility. We have the following.

Proposition 5 Let $\Phi$ and $\phi$ denote, respectively, the standard Gaussian cumulative distribution and density function. In the Black-Scholes model, the CoCo's price sensitivity to share price $\Delta:=\frac{\partial \pi_{t}}{\partial S_{t}}$ and to volatility $v:=\frac{\partial \pi_{t}}{\partial \sigma}$ are respectively given by

$$
\begin{aligned}
\Delta= & \sum_{j: T_{j}>t}^{m} \mathbf{1}_{\left\{t<\tau_{j}\right\}} \frac{c_{j}}{L_{j}}\left(\frac{2}{\sigma \sqrt{T_{j}-t}} \phi\left(b_{-}^{j}\right)-\Phi\left(b_{-}^{j}\right)\right) \\
& +\left(\frac{K}{L_{m}}-C_{r}\right)\left(\frac{2}{\sigma \sqrt{T_{m}-t}} \phi\left(b_{-}^{m}\right)-\Phi\left(b_{-}^{m}\right)\right),
\end{aligned}
$$

and

$$
\begin{aligned}
v= & \sum_{j: T_{j}>t}^{m} \mathbf{1}_{\left\{t<\tau_{j}\right\}} \frac{c_{j}}{L_{j}} \frac{\log \frac{L_{j}}{S_{t}}-r\left(T_{j}-t\right)}{\sigma^{2} \sqrt{T_{j}-t}}\left(\phi\left(-b_{+}^{j}\right) L_{j} \mathrm{e}^{-r\left(T_{j}-t\right)}+S_{t} \phi\left(b_{-}^{j}\right)\right) \\
& -\left(C_{r}-\frac{K}{L_{m}}\right) \frac{\log \frac{L_{m}}{S_{t}}-r\left(T_{m}-t\right)}{\sigma^{2} \sqrt{T_{j}-t}}\left(\phi\left(b_{+}^{m}\right) L_{m} \mathrm{e}^{-r\left(T_{m}-t\right)}+S_{t} \phi\left(b_{-}^{m}\right)\right),
\end{aligned}
$$

where

$$
b_{ \pm}^{j}=\frac{\log \frac{L_{j}}{S_{t}}-\left(r \mp \frac{1}{2} \sigma^{2}\right)\left(T_{j}-t\right)}{\sigma \sqrt{T_{j}-t}}, \quad j=1, \ldots, m
$$


Some remarks are in order. On the one hand, it is has been documented that by actively hedging the equity risk, investors can unintentionally force the conversion by making the share price deteriorate and eventually trigger the conversion. This situation is referred to as the death-spiral effect. Now, from the above expression for the Delta $\Delta$ it can be checked that it is strictly positive, and in fact one can observe that the Delta $\Delta$ increases sharply when the time to maturity $T$ decreases. Thus one of the conclusions in [13] is that the coupon cancellation feature leads to a flatter behaviour of the Delta $\Delta$, hence reducing the death-spiral risk. On the other hand, it can also be checked that the Vega $v$ is strictly negative, this tell us that an increase in the volatility translates into a decrease in the prices. Such observation is clearly in line with the intuition that a higher volatility will increase the probability of crossing the barriers $\ell$ and $\ell^{j}$ defining the conversion and coupon cancellation events.

\section{Advanced Models}

\subsection{Incorporating the Heston Stochastic Volatility Model}

Let us start this section by remarking the fact that the arguments preceding the obtainment of Proposition 4 hold even if the share and default-free bond price volatilities (i.e., $\sigma$ and $b\left(\cdot, T_{j}\right)$ in (17) and (16)) are no longer deterministic. However, the time change $a_{j}$ in (20) would be now stochastic and, consequently, the time-changed fundamental process dynamics

$$
\mathrm{d} U_{a_{j}(t)}^{j}=-\frac{1}{2} a_{j}(t) \mathrm{d} t+\mathrm{d} W_{a_{j}(t)}^{T_{j}}
$$

would no longer match those of a drifted Brownian motion. Thus one anticipates that the pricing problem, in the setting of stochastic volatility, will lead to closed-form formulas only in few cases, and require more advanced numerical tools otherwise.

As a particular model, we shall assume that the volatilities are stochastic according to the work of [26]: we consider a new stochastic factor $\left(V_{t}\right)_{t \geq 0}$ acting on both $\sigma$ and $b\left(\cdot, T_{j}\right)$, in such a way that the dynamics in (17) and (16) are now replaced by

$$
\frac{\mathrm{d} S_{t}}{S_{t}}=\left[r_{t}-\kappa(t)\right] \mathrm{d} t+\sqrt{V_{t}} \sum_{k=1}^{d} \sigma_{k}(t) \mathrm{d} W_{t}^{k},
$$

and

$$
\frac{\mathrm{d} B\left(t, T_{j}\right)}{B\left(t, T_{j}\right)}=r_{t} \mathrm{~d} t+\sqrt{V_{t}} \sum_{k=1}^{d} b_{k}\left(t, T_{k}\right) \mathrm{d} W_{t}^{k}
$$


respectively. The factor $\left(V_{t}\right)_{t \geq 0}$ is given as the solution to the following $\operatorname{SDE}$

$$
\mathrm{d} V_{t}=\left[\alpha-\beta V_{t}\right] \mathrm{d} t+\gamma \sqrt{V_{t}} \mathrm{~d} Z_{t},
$$

where $\alpha, \beta$ and $\gamma$ are constants, with $2 \alpha>\gamma^{2}$ to ensure the positivity of the solution, and $\left(Z_{t}\right)_{t \geq 0}$ is a one-dimensional $\mathbb{P}^{T_{j}}$-Brownian motion. Similar dynamics under the share measure $\mathbb{P}^{(S)}$ are assumed to be satisfied by $\left(V_{t}\right)_{t \geq 0}$.

If we further assume the independence between the noises driving the prices and their volatilities, then we see that the $\mathbb{P}^{T_{j}}$-Brownian motion in (24) is independent of the (now stochastic) time change

$$
a_{j}(t)=\int_{0}^{t} V_{s}\left\|\sigma(s)-b\left(s, T_{j}\right)\right\|^{2} \mathrm{~d} s, \quad 0 \leq t \leq T_{j} .
$$

Thus, by a preconditioning argument, we obtain the following extension of Proposition 4.

Proposition 6 In the current setting, the CoCo arbitrage-free price, on the set $\{t<$ $\left.\tau_{m}\right\}$, is given by

$$
\begin{aligned}
& \pi_{t}=\sum_{j, T_{j}>t}^{m} \mathbf{1}_{\left\{t<\tau_{j}\right\}} c_{j}\left(B\left(t, T_{j}\right) \mathbb{E}^{T_{j}}\left[\Phi\left(-d_{+}^{j}-D_{j}\right) \mid \mathscr{F}_{t}\right]-\frac{S_{t} \mathbb{E}^{T_{j}}\left[\Phi\left(d_{-}^{j}-D_{j}\right) \mid \mathscr{F}_{t}\right]}{L_{j} \mathrm{e} \int_{t}^{T_{j}} \kappa(u) \mathrm{d} u}\right) \\
& +K B\left(t, T_{m}\right) \mathbb{E}^{T_{m}}\left[\Phi\left(-d_{-}^{m}\right) \mid \mathscr{F}_{t}\right]-\frac{K S_{t}}{L_{j} \int_{t}^{\int_{t}^{T_{t}}} \kappa(u) \mathrm{d} u} \mathbb{E}^{T_{m}}\left[\Phi\left(d_{+}^{m}\right) \mid \mathscr{F}_{t}\right]
\end{aligned}
$$

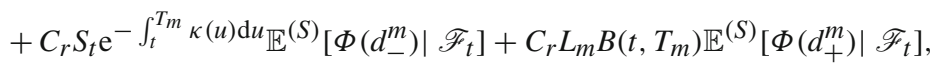

where

$$
d_{ \pm}^{j}=\frac{\log \frac{L_{j} B\left(t, T_{j}\right) \mathrm{e}_{t}^{T_{j}}{ }^{T_{k}}(s) \mathrm{d} s}{S_{t}} \pm \frac{1}{2} \int_{t}^{T_{m}} V_{s}\left\|\sigma(s)-b\left(s, T_{j}\right)\right\|^{2} \mathrm{~d} s}{\sqrt{\int_{t}^{T_{j}} V_{s}\left\|\sigma(s)-b\left(s, T_{j}\right)\right\|^{2} \mathrm{~d} s}}
$$

and

$$
D_{j}=\frac{1}{\sqrt{\int_{t}^{T_{j}} V_{s}\left\|\sigma(s)-b\left(s, T_{j}\right)\right\|^{2} \mathrm{~d} s}} \log \frac{M_{j}}{L_{j}} .
$$

From the pricing formula above we can see that the CoCo price is related to the price of binary options. Indeed, for instance, for a binary option with maturity $T_{j}$ and strike $M_{j}$ we have 


$$
\begin{aligned}
\mathbb{E}^{*}\left[\mathrm{e}^{-\int_{t}^{T_{j}} r_{u} \mathrm{~d} u} \mathbf{1}_{\left\{S_{T_{j}}<M_{j}\right\}} \mid \mathscr{F}_{t}\right] & =B\left(t, T_{j}\right) \mathbb{E}^{T_{j}}\left[\mathbf{1}_{\left\{S_{T_{j}}<M_{j}\right\}} \mid \mathscr{F}_{t}\right] \\
& =B\left(t, T_{j}\right) \mathbb{P}^{T_{j}}\left(U_{T_{j}}^{j} \leq \log \frac{M_{j}}{L_{j}} \mid \mathscr{F}_{t}\right) .
\end{aligned}
$$

Moreover, according to our previous discussion, conditioned to $\mathscr{F}_{T_{j}}^{V}:=\sigma\left(V_{s}, 0 \leq\right.$ $s \leq T_{j}$ ) the random variable $U_{T_{j}}^{j}$ is normally distributed, so that we also have

$$
\mathbb{P}^{T_{j}}\left(U_{T_{j}}^{j} \leq \log \frac{M_{j}}{L_{j}} \mid \mathscr{F}_{t}\right)=\mathbb{E}^{T_{j}}\left[\Phi\left(d_{+}^{j}+D_{j}\right) \mid \mathscr{F}_{t}\right]
$$

Let us now give an explicit computation of the probability above; this illustrates how the CoCo price can be computed. For this matter, we use the relationship between the characteristic and distribution functions (see for instance [38]) which allows us to write

$$
\begin{aligned}
\mathbb{P}^{T_{j}}\left(U_{T_{j}}^{j} \leq \log \frac{M_{j}}{L_{j}} \mid \mathscr{F}_{t}\right)= & \frac{1}{2}+\frac{1}{2 \pi} \\
& \int_{0}^{\infty} \frac{\left(\frac{M_{j}}{L_{j}}\right)^{\mathrm{i} \xi} \varphi^{T_{j}}\left(t, U_{t}, V_{t} ;-\xi\right)-\left(\frac{M_{j}}{L_{j}}\right)^{-\mathrm{i} \xi} \varphi^{T_{j}}\left(t, U_{t}, V_{t} ; \xi\right)}{\mathrm{i} \xi} \mathrm{d} \xi,
\end{aligned}
$$

where

$$
\varphi^{T_{j}}\left(t, U_{t}, V_{t} ; \xi\right):=\mathbb{E}^{T_{j}}\left[\exp \left\{i \xi\left(U_{T_{j}}^{j}\right)\right\} \mid \mathscr{F}_{t}\right]=\mathbb{E}^{T_{j}}\left[\exp \left\{i \xi\left(U_{T_{j}}^{j}\right)\right\} \mid U_{t}, V_{t}\right]
$$

and the last equation holds by Markovianity. Hence the problem of computing the probability above translates into the problem of finding an expression for $\varphi^{T_{j}}(t, u, v ; \xi)$. It follows from the Itô formula that

$$
\frac{\partial \varphi^{T_{j}}}{\partial t}+\frac{1}{2} \frac{\partial^{2} \varphi^{T_{j}}}{\partial u^{2}} v \sigma_{T_{j}}^{2}+\frac{1}{2} \frac{\partial^{2} \varphi^{T_{j}}}{\partial v^{2}} \gamma^{2} v-\frac{1}{2} \frac{\partial \varphi^{T_{j}}}{\partial u} v \sigma_{T_{j}}^{2}+\frac{\partial \varphi^{T_{j}}}{\partial v}(\alpha-\beta v)=0
$$

with the boundary condition $\varphi^{T_{j}}\left(T_{j}, u, v ; \xi\right)=\mathrm{e}^{\mathrm{i} \xi u}$, and where $\sigma_{T_{j}}^{2}=\left\|\sigma-b\left(\cdot, T_{j}\right)\right\|^{2}$. For an affine solution like

$$
\varphi^{T_{j}}(t, u, v ; \xi)=\mathrm{e}^{A_{j}\left(T_{j}-t\right)+B_{j}\left(T_{j}-t\right) v+\mathrm{i} \xi u},
$$

the PDE in (31) is reduced to the Riccati equation

$$
\left\{\begin{array}{c}
\frac{\partial B_{j}}{\partial t}-\frac{1}{2} \gamma^{2} B_{j}^{2}+\beta B_{j}=-\left(\frac{1}{2} \xi^{2}-\frac{i}{2} \xi\right) \sigma_{T_{j}}^{2} \\
\frac{\partial A_{j}}{\partial t}=\alpha B_{j}
\end{array}\right.
$$


with $A_{j}$ and $B_{j}$ vanishing at $t=T_{j}$. As shown in [13], for a constant function $\sigma_{T_{j}}^{2}$, such equation is explicitly solved by

$$
B_{j}\left(T_{j}-t\right)=-\frac{\lambda(\xi)+\beta}{\gamma^{2}} \frac{\exp \left\{-\lambda\left(T_{j}-t\right)\right\}-1}{\exp \left\{-\lambda\left(T_{j}-t\right)\right\}+\frac{\lambda(\xi)+\beta}{\lambda(\xi)-\beta}},
$$

and

$$
\begin{gathered}
A_{j}(t)=-\alpha \frac{\lambda(\xi)-\beta}{\lambda(\xi)+\beta}\left[\frac{2}{\gamma^{2}} \log \left((\lambda(\xi)-\beta) \exp \left\{-\lambda(\xi)\left(T_{j}-t\right)\right\}+\frac{\lambda(\xi)+\beta}{\lambda(\xi)-\beta}\right)\right. \\
\left.+\frac{\lambda(\xi)-\beta}{\gamma^{2}}\left(T_{j}-t\right)\right],
\end{gathered}
$$

where $\lambda(\xi):=\sqrt{\beta^{2}+\gamma^{2} \sigma_{T_{j}}^{2}\left(\xi^{2}-i \xi\right)}$, and $\sqrt{\cdot}$ denote the analytic extension of the real square root to $\mathbb{C} \backslash \mathbb{R}_{-}$.

\subsection{An Exponential Lévy Model}

In this section we shall consider an exponential Lévy model for the share price. As opposed to the previous sections, we shall now consider a numerical approach to pricing, based on exploiting the so-called Wiener-Hopf factorization of the driving Lévy process $\left(X_{t}\right)_{t \geq 0}$. This approach has been recently applied in order to price contracts with path-dependent payoffs as in [12, 31]; see more details below.

\subsubsection{First-Passage Times and Wiener-Hopf Factorization}

Let $\left(X_{t}\right)_{t \geq 0}$ be a Lévy process with characteristic triplet $(\mu, \sigma, v)$, and denote its characteristic exponent by $\psi_{X}$. For details and proofs of the following arguments we refer to [1].

Recall that if $e(\lambda)$ is an exponential random variable with parameter $\lambda$, independent of $\left(X_{t}\right)_{t \geq 0}$, then we have the following equality in distribution

$$
X_{e(\lambda)}=I+S
$$

where $I$ and $S$ are independent random variables, distributed as

$$
\underline{X}_{e(\lambda)}:=\inf _{0 \leq u \leq e(\lambda)} X_{u} \quad \text { and } \quad \bar{X}_{e(\lambda)}:=\sup _{0 \leq u \leq e(\lambda)} X_{u}
$$

respectively. Moreover,

$$
\mathbb{E}\left[\exp \left\{z X_{e(\lambda)}\right\}\right]=\mathbb{E}\left[\exp \left\{z \underline{X}_{e(\lambda)}\right\}\right] \mathbb{E}\left[\exp \left\{z \bar{X}_{e(\lambda)}\right\}\right] .
$$


We shall refer to (34) as the Wiener-Hopf factorization. In fact, in general it holds that

$$
\mathbb{E}\left[\exp \left\{z X_{e(\lambda)}\right\}\right]=\frac{\lambda}{\lambda-\psi_{X}(z)}
$$

Consequently, the knowledge of one of the factors in (34) allows us to establish the other one. A particular case of interest arises when $\left(X_{t}\right)_{t \geq 0}$ is a spectrally negative process since, in this case, it is known that the right factor in (34) is given by

$$
\psi_{\lambda}^{+}(z):=\mathbb{E}\left[\exp \left\{z \bar{X}_{e(\lambda)}\right\}\right]=\frac{\beta_{\lambda}}{\beta_{\lambda}-z},
$$

where $\beta_{\lambda}$ is a constant, depending on $\lambda$, defined as the solution to

$$
\psi_{X}(\beta)=\lambda
$$

Therefore, once we have computed $\beta_{\lambda}$ explicitly, we obtain the following expression for the left factor in (34):

$$
\psi_{\lambda}^{-}(z):=\mathbb{E}\left[\exp \left\{z \underline{X}_{e(\lambda)}\right\}\right]=\frac{\lambda}{\lambda-\psi_{X}(z)} \frac{\beta_{\lambda}-z}{\beta_{\lambda}} .
$$

This expression can be linked to the distribution function of $\underline{X}_{t}$ by partial integration. Indeed we have

$$
\begin{aligned}
\psi_{\lambda}^{-}(z) & =\int_{0}^{\infty} \lambda \mathrm{e}^{-\lambda t} \mathrm{~d} t \int_{-\infty}^{0} z \mathrm{e}^{z \xi} \mathbb{P}\left(\underline{X}_{t}>\xi\right) \mathrm{d} \xi \\
& =\lambda z \int_{0}^{\infty} \int_{0}^{\infty} \mathrm{e}^{-\lambda t-z \xi} F(t, \xi) \mathrm{d} t \mathrm{~d} \xi \\
& =\lambda z \widetilde{F}(\lambda, z)
\end{aligned}
$$

where we have defined $F(t, \xi):=\mathbb{P}\left(\underline{X}_{t}>-\xi\right)$, and denoted its Laplace transform by $\widetilde{F}$. As argued by [33], by combining (37) and (38) we can recover $F$ by the standard Fourier transform inversion. Further, the result can be numerically computed in an efficient way, provided the condition

$$
\lim _{z \rightarrow \infty} \frac{1}{z} \int_{-\infty}^{0}\left[e^{z x}-1-z x \mathbf{1}_{\{|x| \leq 1\}}\right] v(\mathrm{~d} x)=0,
$$

holds true. This condition is imposed in order to facilitate the computation of $\beta_{\lambda}$ (i.e., the solution of (35)) by means of suitable integration contour change. In fact, such a change allows to take $\beta_{\lambda}=\lambda / \mu$. This result is summarized in the following lemma. 
Lemma 7 For fixed $t$ and $\xi$, and given the parameter set $\left(A_{1}, A_{2}, l_{1}, l_{2}, N_{1}, N_{2}\right)$, define

$$
a_{1}:=\frac{A_{1}}{2 t l_{1}}, \quad a_{2}:=\frac{A_{2}}{2 \xi l_{2}}, \quad h_{1}:=\frac{\pi}{t l_{1}}, \quad h_{2}:=\frac{\pi}{\xi l_{2}}, \quad g(x):=\psi_{X}(x / \mu)
$$

and, for every $N \in \mathbb{N}$,

$$
\begin{aligned}
s_{N}(t, \xi):= & \frac{h_{1} h_{2}}{4 \pi^{2}} \sum_{n=-N}^{N} \sum_{k=-N}^{N} \frac{\mathrm{d} g}{\mathrm{~d} x}\left(a_{1}+\mathrm{i} n h_{1}\right) \\
& \widetilde{F}\left(g\left(a_{1}+\mathrm{i} n h_{1}\right), a_{2}+\mathrm{i} k h_{2}\right) \mathrm{e}^{t g\left(a_{1} t+\mathrm{i} n h_{1}\right)+x\left(a_{2}+\mathrm{i} k h_{2}\right)} .
\end{aligned}
$$

If the condition (39) is satisfied, then the following approximation holds true

$$
\mathbb{P}\left(\underline{X}_{t}>\xi\right) \doteqdot \sum_{n=0}^{N_{2}}\left(\begin{array}{c}
N_{2} \\
n
\end{array}\right) s_{N_{1}+n}(t, \xi)
$$

where the symbol $\doteqdot$ indicates an Euler summation.

The double sum in the lemma is used as an initial approximation of $F$. Here the parameters $\left(A_{1}, A_{2}, l_{1}, l_{2}\right)$ are positive real numbers chosen large enough in order to control the aliasing error. The final Euler summation is used in order to improve the accuracy of the raw approximation $s_{N}$. It is suggested that choosing $A_{1}=A_{2}=22$, $l_{1}=l_{2}=1, N_{1}=12$ and $N_{2}=15$ gives satisfactory results. For further details see [33] and references therein.

\subsubsection{The One-Sided CGMY Lévy Process}

Hereafter we shall focus on a particular spectrally negative Lévy process $\left(X_{t}\right)_{t \geq 0}$ known as one-sided CGMY process, or simply $C M Y$ process. This process has no continuous part, and only one-sided jumps with its Lévy measure being given by

$$
v(\mathrm{~d} x)=C \exp \{-M x\}|x|^{-1-Y} \mathbf{1}_{\{x<0\}} \mathrm{d} x,
$$

where $C, M>0$ and $Y<1$ are constants. Further, its characteristic exponent can be obtained in closed-form as

$$
\begin{aligned}
\psi_{X}(z) & =\mu z+\int_{-\infty}^{0}\left[e^{z x}-1-z x \mathbf{1}_{\{|x| \leq 1\}}\right] v(\mathrm{~d} x) \\
& =\mu z+C \Gamma(-Y)\left((M+z)^{Y}-M^{Y}\right)
\end{aligned}
$$


where $\Gamma$ stands for the Gamma function. Thus it is apparent that the condition (39) holds true. Before we proceed, let us mention that the CGMY processes are also referred to as Tempered stable processes. On the other hand, by setting the parameter $Y=0$ (resp., $Y=1 / 2$ ) the CMY process becomes a Gamma process (resp., Inverse Gaussian process). For details on CMY processes we refer to [4, 31] and [36, Section 2.3.5].

In order to price CoCos we need to understand the behavior of $\left(X_{t}\right)_{t \geq 0}$ both under the risk-neutral measure $\mathbb{P}^{*}$ and the share measure $\mathbb{P}^{(S)}$. The following result shows how the Lévy characteristics of $\left(X_{t}\right)_{t \geq 0}$ change under Esscher transforms.

Lemma 8 For every real number $\theta$, consider the probability measure $\mathbb{P}^{\alpha}$, equivalent to $\mathbb{P}$, given by

$$
\frac{\mathrm{d} \mathbb{P}^{\alpha}}{\mathrm{dP}}=\frac{\exp \left\{\alpha X_{T}\right\}}{\mathbb{E}\left[\exp \left\{\alpha X_{T}\right\}\right]}
$$

Assume $M_{\alpha}:=M-\alpha>0$. Then the Lévy exponent of $\left(X_{t}\right)_{t \geq 0}$ under $\mathbb{P}^{\alpha}$ is given by

$$
\psi_{X}^{\alpha}(z):=z \mu_{\alpha}+\int_{-\infty}^{0}\left[e^{z x}-1-z x \mathbf{1}_{\{|x| \leq 1\}}\right] v_{\alpha}(\mathrm{d} x)
$$

where

$$
\mu_{\alpha}:=\mu+\int_{|x| \leq 1} x\left(e^{\alpha x}-1\right) v(\mathrm{~d} x), \quad \text { and } \quad v_{\alpha}(\mathrm{d} x):=C \exp \left\{-M_{\alpha} x\right\}|x|^{-1-Y} \mathbf{1}_{\{x<0\}} \mathrm{d} x .
$$

Proof The first part follows from (41) and [35, Theorems 33.1 and 33.2]. Now, using the expression in (40) we have

$$
v_{\alpha}(\mathrm{d} x)=C \exp \{-(M-\alpha) x\}|x|^{-1-Y} \mathbf{1}_{\{x<0\}} \mathrm{d} x=C \exp \left\{-M_{\alpha} x\right\}|x|^{-1-Y} \mathbf{1}_{\{x<0\}} \mathrm{d} x .
$$

The assumption $M_{\alpha}>0$ assures that $\left(C, M_{\alpha}, Y\right)$ is a rightful parameter set for a CMY distribution.

\subsubsection{Application to $\operatorname{CoCos}$}

We shall assume that, under $\mathbb{P}^{*}$, there is a pure-jump $(C, M, Y)$-Lévy process $\left(X_{t}\right)_{t \geq 0}$ driving the share price $\left(S_{t}\right)_{t \geq 0}$ in such a way that

$$
S_{t}:=\mathrm{e}^{(r-\kappa) t} \frac{\exp \left\{X_{t}\right\}}{\mathbb{E}^{*}\left[\exp \left\{X_{t}\right\}\right]}=\exp \left\{\mu t+X_{t}\right\}, \quad t \geq 0,
$$

where the interest rate $r$ and the dividends $\kappa$ are assumed to be constants, and we define $\varpi:=-\log \mathbb{E}^{*}\left[\mathrm{e}^{X_{1}}\right]$ and set $\mu:=r-\kappa+\varpi$. Further, we shall assume that the 
parameter $M$ is bigger than 1 . We remark here that, on the one hand, this technical assumption will allow us to accommodate the Variance Gamma (VG) process considered in [30]. On the other hand, this assumption is consistent with the numerical experiments reported by [31].

Proposition 9 In the current setting, the price of a CoCo at time $0 \leq t \leq T$ can be numerically approximated in an efficient way by means of the expression

$$
\pi_{t} \approx \sum_{j: T_{j}>t}^{m} \mathrm{e}^{-r\left(T_{j}-t\right)} P_{j}^{0}\left(t, T_{j}\right)+K \mathrm{e}^{-r\left(T_{m}-t\right)} P_{m}^{0}\left(t, T_{m}\right)+C_{r} S_{t} \mathrm{e}^{-\kappa\left(T_{m}-t\right)} P_{m}^{1}\left(t, T_{m}\right),
$$

where

$$
P_{j}^{\alpha}\left(t, T_{j}\right) \doteqdot \sum_{n=0}^{N_{2}}\left(\begin{array}{c}
N_{2} \\
n
\end{array}\right) s_{N_{1}+n}^{\alpha}\left(T_{j}-t, \log \frac{S_{t}}{\ell^{j}(t)}+\varpi\left(T_{j}-t\right)\right), \quad j=1, \ldots, m, \alpha=0,1,
$$

with the symbol $\doteqdot$ indicating an Euler summation, and

$$
\begin{aligned}
& s_{N}^{\alpha}(t, \xi):=\sum_{n=-N}^{N} \sum_{k=-N}^{N} \frac{\mu_{\alpha}+Y C \Gamma(-Y)\left(M_{\alpha}+\mu_{\alpha}^{-1} t\right)^{Y-1}}{4 \mu_{\alpha} t \xi l_{1} l_{2}} \\
& \widetilde{F}_{\alpha}\left(g_{\alpha}\left(a_{1}+\mathrm{i} n h_{1}\right), a_{2}+\mathrm{i} k h_{2}\right) \mathrm{e}^{t g_{\alpha}\left(a_{1} t+\mathrm{i} n h_{1}\right)+x\left(a_{2}+\mathrm{i} k h_{2}\right)},
\end{aligned}
$$

with the parameters $\left(a_{1}, a_{2}, h_{1}, h_{2}, l_{1}, l_{2}, N_{1}, N_{2}\right)$ given as in Lemma 7 , and

$$
\begin{gathered}
\widetilde{F}_{\alpha}(\lambda, z):=\frac{\lambda-z \mu_{\alpha}}{\left(\lambda-z \mu_{\alpha}-C \Gamma(-Y)\left(\left(M_{\alpha}+z\right)^{Y}-M_{\alpha}^{Y}\right)\right) z \lambda}, \\
g_{\alpha}(x):=x+C \Gamma(-Y)\left(\left(M_{\alpha}+\mu_{\alpha}^{-1} x\right)^{Y}-M_{\alpha}^{Y}\right), \quad \alpha=0,1,
\end{gathered}
$$

where $M_{\alpha}$ and $\mu_{\alpha}$ are defined in Lemma 8.

Proof Taking into account the general expression for the CoCo price (c.f. Proposition 2 ), computing $\pi_{t}$ boils down to compute

$$
P_{j}^{0}\left(t, T_{j}\right):=\mathbb{P}^{*}\left(\tau_{j}>T_{j} \mid \mathscr{F}_{t}\right)=\left.\mathbb{P}^{*}\left(\underline{\mathrm{X}}_{T_{j}-t}>-\xi\right)\right|_{\xi=\log \left(S_{t} / \ell^{j}(t)\right)+\varpi\left(T_{j}-t\right)},
$$

for $j=1, \ldots, m$, and

$$
P_{m}^{1}\left(t, T_{m}\right):=\mathbb{P}^{(S)}\left(\tau_{m}>T_{m} \mid \mathscr{F}_{t}\right)=\left.\mathbb{P}^{(S)}\left(\underline{X}_{T_{m}-t}>-\xi\right)\right|_{\xi=\log \left(S_{t} / \ell^{m}(t)\right)+\varpi\left(T_{m}-t\right)} .
$$

These computations can be carried out by means of Lemma 7. Indeed, under this exponential Lévy model, the share measure $\mathbb{P}^{(S)}$ (resp., risk-neutral measure $\mathbb{P}^{*}$ ) 
coincides with an Esscher transform of parameter $\alpha=1$ (resp., $\alpha=0$ ). In light of Lemma 8 , the driving noise $\left(X_{t}\right)_{t \geq 0}$ will remain a CMY process under $\mathbb{P}^{(S)}$, but now having the shifted parameter set $\left(C, M_{1}, Y\right)=(C, M-1, Y)$. Moreover, this implies that the Lévy measure of $\left(X_{t}\right)_{t>0}$ also satisfies the condition (39) under $\mathbb{P}^{(S)}$. Thus, if we write $\mathbb{P}^{0}=\mathbb{P}^{*}$ and $\mathbb{P}^{1}=\mathbb{P}^{(S)}$, by the reasoning in Sect. 4.2.1 we see that the Laplace transform of $F_{\alpha}(t, \xi):=\mathbb{P}^{\alpha}\left(\underline{X}_{t}>-\xi\right)$ is given by $\widetilde{F}_{\alpha}$ as defined above. Moreover, the correspondent contour change is given by $g_{\alpha}$.

Remark 10 [12] provides an alternative approach which exploits the Wiener-Hopf factorization in a different way: instead of computing first-time passage probabilities as done here, what is computed is the joint density of $\left(X_{t}, \underline{X}_{t}\right)$. As the noise driving share prices, the authors consider the so-called Beta-Variance Gamma ( $\beta$ VG) process_-also referred to as Lamperti-Stable process by [3] — which exhibits the same exponential decay as the Variance Gamma process, hence leading to a smileconform model. For this $\beta$-VG process the distribution of the variables $\bar{X}_{e(\lambda)}$ and $\underline{X}_{e(\lambda)}$ can be specified, thus obtaining the Wiener-Hopf factors $\psi_{\lambda}^{+}$and $\psi_{\lambda}^{-}$. Taking (8) into account, combining the knowledge of the $\left(X_{t}, \underline{X}_{t}\right)$ density with a MonteCarlo technique due to [29], the authors provide an efficient numerical pricing of CoCos.

\section{Triggering Conversion Under Short-Term Uncertainty}

Linking credit events to the movements of a fully observable (i.e., $\mathbb{F}$-adapted) process $\left(U_{t}\right)_{t \geq 0}$ is certainly one of the most appealing features of structural models. Indeed, this full observability assumption - hereafter referred to as (A1) - gives rise to clear and analytically tractable models as we have seen in the previous sections. When considering contingent capital contracts such as CoCos, however, the assumption (A1) seems arguable since in most cases regulatory capital depends on the balance sheets of the issuer, and those sheets are updated only at a series of predetermined dates $\left(t_{j}\right)_{t \in \mathbb{N}}$. Thus we are interested in considering the following partial observability assumption.

Assumption $\left(\mathbf{A} \mathbf{1}^{\prime}\right)$. The fundamental process $\left(U_{t}\right)_{t \geq 0}$ is fully observable only at predetermined dates $\left(t_{j}\right)_{t \in \mathbb{N}}$.

On the other hand, when the process $\left(U_{t}\right)_{t \geq 0}$ is related to the share price, it is also commonly assumed that the correlation between the noises driving the share price and $\left(U_{t}\right)_{t \geq 0}$ is equal to $\rho=1$ (or $\rho=-1$ ) - hereafter this assumption will be referred to as (A2). Nevertheless, it would be reasonable to consider the chance that a different (possibly time-dependent) correlation parameter $\rho \in[-1,1]$ may provide a better fit. Consequently we are also interested in considering the correlation $\rho$ as an additional rightful model parameter by taking the following alternative to (A2).

Assumption $\left(\mathbf{A 2}^{\prime}\right)$. The correlation $\rho$ between the noises driving the share price and $\left(U_{t}\right)_{t \geq 0}$ may vary. 
In what follows we revisit the simple framework of Sect. 3.1 in order to illustrate these ideas and show how the pricing problem is modified under $\left(\mathbf{A} \mathbf{1}^{\prime}\right)$ and $\left(\mathbf{A} \mathbf{2}^{\prime}\right)$. For a full study of this short-term uncertainty model we refer to the forthcoming paper [15].

\subsection{Pricing CoCos on a Black-Scholes Model Under Short-term Uncertainty}

As shown in Sect. 3.1, in the Black-Scholes model,

$$
\mathrm{d} S_{t}=S_{t}\left([r-\kappa] \mathrm{d} t+\sigma \mathrm{d} W_{t}^{*}\right)
$$

the cancellation of the $j$-th coupon is triggered as soon as the process

$$
\mathrm{d} U_{t}:=\mathrm{d} \log \frac{S_{t}}{\ell_{t}}=-\frac{1}{2} \sigma^{2} \mathrm{~d} t+\sigma \mathrm{d} W_{t}^{*},
$$

crosses the critical value $\log \frac{M_{j}}{L_{j}}, j=1, \ldots, m$, whereas for conversion zero is the critical level. In this setting Assumption (A2 $\left.{ }^{\prime}\right)$ is translated as the correlation structure between the noise driving $\left(S_{t}\right)_{t \geq 0}$ and that of the new process

$$
\mathrm{d} U_{t}(\rho):=-\frac{1}{2} \sigma^{2} \mathrm{~d} t+\sigma \mathrm{d} W_{t}^{\rho}:=-\frac{1}{2} \sigma^{2} \mathrm{~d} t+\sigma \mathrm{d}\left(\rho W_{t}^{*}+\sqrt{1-\rho^{2}} Z_{t}\right),
$$

where $\rho$ is the given correlation parameter, and $\left(Z_{t}\right)_{t \geq 0}$ is a second Brownian motion, independent of $\left(W_{t}^{*}\right)_{t \geq 0}$. Thus, instead of the process $\left(U_{t}\right)_{t \geq 0}$ above, we shall now consider the parametric family $\left(U_{t}(\rho)\right)_{t \geq 0}$ whose driving noise $\left(W_{t}^{\rho}\right)_{t \geq 0}$ is also a Brownian motion but correlated to $\left(W_{t}^{*}\right)_{t \geq 0}$, in such a way that $\mathrm{d} W_{t}^{\rho} \mathrm{d} W_{t}^{*}=\rho \mathrm{d} t$. Further, the time at which the $j$-th coupon may be cancelled is given by

$$
\tau_{j}(\rho):=\inf \left\{t \geq 0: U_{t}(\rho) \leq \log \frac{M_{j}}{L_{j}}\right\} .
$$

As for Assumption $\left(\mathbf{A} \mathbf{1}^{\prime}\right)$, notice that the full information flow corresponds to

$$
\mathscr{G}_{t}:=\sigma\left(W_{s}^{*}, Z_{s}, 0 \leq s \leq t\right)=\mathscr{F}_{t}^{W^{*}} \vee \mathscr{F}_{t}^{Z}, \quad t \geq 0,
$$

whereas, setting $\lfloor t\rfloor:=\min \left\{t_{j} \in\left\{0, t_{1}, t_{2}, \ldots\right\} \quad: t_{j} \leq t<t_{j+1}\right\}$, the information available to the modeller is now given by

$$
\widetilde{\mathscr{F}}_{t}:=\mathscr{F}_{t}^{W^{*}} \vee \sigma\left(Z_{s}, 0 \leq s \leq\lfloor t\rfloor\right)=\mathscr{F}_{t}^{W^{*}} \vee \mathscr{F}_{\lfloor t\rfloor}^{Z}, \quad t \geq 0 .
$$


Since $\mathscr{G}_{t} \supseteq \widetilde{\mathscr{F}}_{t}$, and the equality holds only at the predetermined dates $\left\{t_{j}, j \in \mathbb{N}\right\}$, we can think of $\left(Z_{t}\right)_{t \geq 0}$ as an extra source of noise which clears out at update times $\left(t_{j}\right)_{j \in \mathbb{N}}$. The fact that the extra noise is cleared out at $\left(t_{j}\right)_{j \in \mathbb{N}}$ motivates the notion of short-term uncertainty, and it has two important implications. On the one hand, our model differs from other partial or incomplete information models like [9, 10, 21], or [25] since the information structure is different. On the other hand, as opposed to other structural models, the short-term uncertainty considered here prevents the conversion time $\tau_{j}(\rho)$ from being a stopping time with respect to the reference filtration $\mathbb{F}$, which is generated by the relevant state variables and the risk-free market. Hence, one can investigate conditions under which $\tau_{j}(\rho)$ admits an intensity, as done by $[9,21]$, or [27].

\subsection{Coupon Cancellation Probabilities Under Short-Term Uncertainty}

Let us show how the coupon cancellation probabilities are modified under the assumptions $\left(\mathbf{A} \mathbf{1}^{\prime}\right)$ and $\left(\mathbf{A} \mathbf{2}^{\prime}\right)$. We begin by defining two auxiliary processes

$$
\zeta_{t}:=\sigma \sqrt{1-\rho^{2}}\left(Z_{t}-Z_{\lfloor t\rfloor}\right) \quad \text { and } \quad \xi_{t}:=\rho \log \frac{S_{t}}{S_{\lfloor t\rfloor}}+\rho \log \frac{\ell_{t}}{\ell_{\lfloor t\rfloor}}, \quad t \geq 0 .
$$

These processes have an important role in the computations within our short-term uncertainty model since they appear implicitly in $\left(U_{t}(\rho)\right)_{t \geq 0}$ according to the factorization

$$
U_{t}(\rho)=\left(U_{\lfloor t\rfloor}(\rho)+\xi_{t}\right)+\zeta_{t}
$$

It is apparent that the term between parentheses belongs to $\widetilde{\mathscr{F}}_{t}=\mathscr{F}_{t}^{W^{*}} \vee \mathscr{F}_{\lfloor t\rfloor}^{Z}$. On the other hand, $\zeta_{t}$ is independent of $\widetilde{\mathscr{F}}_{t}$, and it is normally distributed with zero mean and variance

$$
v^{2}(t):=\left(1-\rho^{2}\right)(t-\lfloor t\rfloor) \sigma^{2}
$$

We note here that the variance $v^{2}(t)$ represents a key quantity within this framework. Indeed, on the one hand, it actually encodes the two new features of our model: the factor $1-\rho^{2}$ measures how close $\left(S_{t}\right)_{t \geq 0}$ and $\left(U_{t}\right)_{t \geq 0}$ are to being completely correlated; whereas the factor $t-\lfloor t\rfloor$ measures the elapsed time from the last information update. On the other hand, as the following result suggests, coupon cancellation probabilities and other analytical formulas obtained within our model depend explicitly on $v(t)$. 
Proposition 11 For every $x \in \mathbb{R}$ define the random time $\tau_{x}(\rho):=\inf \left\{s \geq 0: U_{s}\right.$ $(\rho) \leq x\}$. Then, for every $0 \leq t \leq T$, the following equation holds true on $\{\tau>\lfloor t\rfloor\}$

$$
\begin{aligned}
\mathbb{P}^{*}\left(\tau_{x}(\rho)>T \mid \widetilde{\mathscr{F}}_{t}\right)= & \mathbb{E}^{*}\left[\Phi\left(-D_{-}+\frac{\zeta_{t}}{\sigma \sqrt{T-t}}\right)\right]-\mathrm{e}^{-\left(U_{\lfloor t\rfloor}(\rho)-x\right)}\left(\frac{S_{\lfloor t\rfloor} \ell_{\lfloor t\rfloor}}{S_{t} \ell_{t}}\right) \\
& \mathbb{E}^{*}\left[\mathrm{e}^{-\zeta_{t}} \Phi\left(D_{+}-\frac{\zeta_{t}}{\sigma \sqrt{T-t}}\right)\right],
\end{aligned}
$$

where the expectations above are restricted to the values

$$
D_{ \pm}=\frac{x-U_{\lfloor t\rfloor}(\rho)-\xi_{t} \pm \frac{1}{2} \sigma^{2}(T-t)}{\sigma \sqrt{T-t}}
$$

Moreover,

$$
\mathbb{E}^{*}\left[\Phi\left(-D_{-}+\frac{\zeta_{t}}{\sigma \sqrt{T-t}}\right)\right]=\int_{\mathbb{R}} \Phi\left(-D_{-}+\frac{z v(t)}{\sigma \sqrt{T-t}}\right) \phi(z) \mathrm{d} z
$$

and

$$
\mathbb{E}^{*}\left[\mathrm{e}^{-\zeta_{t}} \Phi\left(D_{+}-\frac{\zeta_{t}}{\sigma \sqrt{T-t}}\right)\right]=\mathrm{e}^{\frac{1}{2} v^{2}(t)} \int_{\mathbb{R}} \Phi\left(D_{+}-\frac{z v(t)-v^{2}(t)}{\sigma \sqrt{T-t}}\right) \phi(z) \mathrm{d} z,
$$

where $\Phi$ and $\phi$ stand, respectively, for the standard Gaussian cumulative distribution and density functions.

Proof Since under $\mathscr{G}_{t}$ the computation is known, for every $t=t_{j}$ we have, on $\left\{\tau_{x}(\rho)>t\right\}$

$$
\begin{aligned}
\mathbb{P}^{*}\left(\tau_{x}(\rho)>T \mid \widetilde{\mathscr{F}}_{t_{j}}\right)= & \mathbb{P}^{*}\left(\tau_{x}(\rho)>T \mid \mathscr{G}_{t_{j}}\right) \\
= & \Phi\left(\frac{-x+U_{t_{j}}(\rho)+\frac{1}{2} \sigma^{2}\left(T-t_{j}\right)}{\sigma \sqrt{T-t_{j}}}\right)-\mathrm{e}^{-\left(U_{t_{j}}(\rho)-x\right)} \Phi \\
& \left(\frac{x-U_{t_{j}}(\rho)+\frac{1}{2} \sigma^{2}\left(T-t_{j}\right)}{\sigma \sqrt{T-t_{j}}}\right)
\end{aligned}
$$

Define

$$
d_{ \pm}^{1}(t)=\frac{x-U_{t}(\rho) \pm \frac{1}{2} \sigma^{2}(T-t)}{\sigma \sqrt{T-t}}
$$


For $t \notin\left\{t_{j}, j \in \mathbb{N}\right\}$, by conditioning we get, on $\left\{\tau_{x}(\rho)>\lfloor t\rfloor\right\}$,

$$
\begin{aligned}
\mathbb{P}^{*}\left(\tau_{x}(\rho)>T \mid \widetilde{\mathscr{F}}_{t}\right)= & \mathbb{E}^{*}\left[\mathbb{P}^{*}\left(\tau_{x}(\rho)>T \mid \mathscr{G}_{t}\right) \mid \widetilde{\mathscr{F}}_{t}\right]=\mathbb{E}^{*}\left[\Phi\left(-d_{-}^{1}(t)\right) \mid \widetilde{\mathscr{F}}_{t}\right] \\
& -\mathbb{E}^{*}\left[\mathrm{e}^{-\left(U_{t}(\rho)-x\right)} \Phi\left(d_{+}^{1}(t)\right) \mid \widetilde{\mathscr{F}}_{t}\right] .
\end{aligned}
$$

In these terms, for $\lfloor t\rfloor<t$, the first summand above satisfies

$$
\mathbb{E}^{*}\left[\Phi\left(-d_{-}(t)\right) \mid \widetilde{\mathscr{F}}_{t}\right]=\mathbb{E}^{*}\left[\Phi\left(-D_{-}+\frac{\zeta_{t}}{\sigma \sqrt{T-t}}\right)\right],
$$

where the right-hand side of the expectation above is restricted to the value of $D_{-}$ given in (45); in fact the equation above reduces to (46) since $\zeta_{t} \sim N\left(0, v^{2}(t)\right)$ for every fixed $t \geq 0$. Similarly for the second summand, it follows from (44) that

$$
\begin{aligned}
\mathbb{E}^{*}\left[\mathrm{e}^{-\left(U_{t}(\rho)-x\right)} \Phi\left(d_{+}^{1}\right) \mid \widetilde{\mathscr{F}}_{t}\right] & =\mathrm{e}^{-\left(U_{\lfloor t\rfloor}(\rho)-x+\xi_{t}\right)} \mathbb{E}^{*}\left[\mathrm{e}^{-\zeta_{t}} \Phi\left(D_{+}-\frac{\zeta_{t}}{\sigma \sqrt{T-t}}\right)\right] \\
& =\mathrm{e}^{-\left(U_{\lfloor t\rfloor}(\rho)-x\right)}\left(\frac{S_{\lfloor t\rfloor} \ell_{\lfloor t\rfloor}}{S_{t} \ell_{t}}\right) \mathbb{E}^{*}\left[\mathrm{e}^{-\zeta_{t}} \Phi\left(D_{+}-\frac{\zeta_{t}}{\sigma \sqrt{T-t}}\right)\right] .
\end{aligned}
$$

In order to obtain (47), let us consider the change of measure given by

$$
\begin{aligned}
\frac{\mathrm{dP}^{\prime}}{\mathrm{d} \mathbb{P}^{*}}=\exp \left\{\zeta_{t}-\frac{1}{2} v^{2}(t)\right\} & =\exp \left\{\int_{0}^{T} \sigma \sqrt{1-\rho^{2}} \mathbf{1}_{[\lfloor t\rfloor, t]}(s) \mathrm{d} Z_{s}\right. \\
& \left.-\frac{1}{2} \int_{0}^{T}\left[\sigma \sqrt{1-\rho^{2}} \mathbf{1}_{[\lfloor t\rfloor, t]}(s)\right]^{2} \mathrm{~d} s\right\}
\end{aligned}
$$

In virtue of the Girsanov theorem, the process

$$
Z_{s}^{\prime}:=Z_{s}-\int_{0}^{s} \sigma \sqrt{1-\rho^{2}} \mathbf{1}_{[\lfloor t\rfloor, t]}(u) \mathrm{d} u, \quad s \geq 0,
$$

follows a $\mathbb{P}^{\prime}$-Brownian motion. Thus

$$
\begin{aligned}
\mathbb{E}^{*}\left[\mathrm{e}^{\zeta_{t}-\frac{1}{2} v^{2}(t)} \Phi\left(D_{+}-\frac{\zeta_{t}}{\sigma \sqrt{T-t}}\right)\right] & =\mathbb{E}_{\mathbb{P}^{\prime}}\left[\Phi\left(D_{+}-\frac{\zeta_{t}}{\sigma \sqrt{T-t}}\right)\right] \\
& =\mathbb{E}_{\mathbb{P}^{\prime}}\left[\Phi\left(D_{+}-\frac{\int_{0}^{T} \sigma \sqrt{1-\rho^{2}} \mathbf{1}_{[\lfloor t\rfloor, t]}(s) \mathrm{d} Z_{s}^{\prime}+v^{2}(t)}{\sigma \sqrt{T-t}}\right)\right] \\
& =\int_{\mathbb{R}} \Phi\left(D_{+}-\frac{x+v^{2}(t)}{\sigma \sqrt{T-t}}\right) \frac{\exp \left\{-\frac{x^{2}}{2 v^{2}(t)}\right\}}{\sqrt{2 \pi v^{2}(t)}} \mathrm{d} x \\
& =\int_{\mathbb{R}} \Phi\left(D_{+}-\frac{v(t) z+v^{2}(t)}{\sigma \sqrt{T-t}}\right) \phi(z) \mathrm{d} z
\end{aligned}
$$


where the first equivalence follows from the abstract Bayes' rule, and for the last equivalence we have simply used the standard change of variables $z=\frac{x}{v(t)}$.

Jeanblanc and Valchev [27] study the role of information on defaultable-bond prices within a Black-Scholes setting, with constant parameters and flat default barrier. In some sense, the short-term uncertainty model considered here can be seen as a bivariate extension of [27]. Despite this analogy, immediate differences arise. For instance, the survival probabilities obtained by [27] are constant between observation dates, i.e., within each interval $\left[t_{j}, t_{j+1}\right)$. Whereas in Proposition 11 these probabilities vary in continuous time. This difference relies on the fact that, event though within each interval $\left[t_{j}, t_{j+1}\right)$ our knowledge of the short-term noise $\zeta_{t}$ is constant, we still fully observe the evolution of $\left(S_{t}\right)_{t \geq 0}$ and all the other $\mathbb{F}$-adapted state variables.

In order to conclude this section, let us recall that in light of our discussion in Sect.3.1, CoCo prices can be obtained in our current setting once we compute expressions of the form

$$
\mathbb{P}^{*}\left(\tau_{j}(\rho)>T_{j}, S_{T_{j}}>L_{j} \mid \widetilde{\mathscr{F}}_{t}\right), \quad \text { and } \quad \mathbb{P}^{(S)}\left(\tau_{m}(\rho)>T_{m}, S_{T_{m}}>L_{m} \mid \widetilde{\mathscr{F}}_{t}\right) .
$$

It is worth noticing that the $\widetilde{\mathscr{F}}_{t}$-conditional joint distribution of $\left(\tau_{j}(\rho), S_{T_{j}}\right)=$ (inf $_{s \leq T_{j}} U_{s}(\rho), S_{T_{j}}$ ) cannot be computed directly from Proposition 11 since the entries of this vector are driven by two different (though correlated) Brownian motions. An additional complication comes from the fact that the current information about one of them might be incomplete. The aforementioned distribution, and full details on the model, can be found in [15].

\section{Extension Risk}

According to the new regulatory Basel III framework, CoCos can be categorised as either belonging to the Additional Tier 1 or Tier 2 capital category. In order to belong to the former class, a CoCo is supposed to have the coupon cancellation feature and, further, no fixed maturity is to be imposed to the contract. Instead, the issuer is entitled to redeem the CoCo at any of the prespecified call times $\left\{T_{i}, i \in \mathbb{N}\right\}$. Moreover, as opposed to the common practice on callable contracts before the 2008 financial crisis, the definition of this contract does not contain any incentive (e.g., a coupon step-up) for the issuer to redeem at the first call date. Investing in such a contract has the inherent risk of a financial loss due to the lengthening of the (investor's) expected maturity duration which ultimately postpones the payment of the face value $K$. This risk is referred to as extension risk. Two recent papers [14, 18] have addressed the problem of pricing CoCos belonging to the Additional Tier 1 capital category. As an illustration, let us revisit the Black-Scholes model in Sect.3.1.

In order to emphasize the correspondence with call times, we add now an extra index $i \in \mathbb{N}$ to the coupon structure, in such a way that a coupon $c_{i j}$ will be paid 
at $T_{i j}$ provided $\tau_{i j}>T_{i j}$. It will be assumed that for every $i \in \mathbb{N}$ the ordering $T_{i-1}<T_{i 1}, \ldots \leq T_{i m}:=T_{i}$ holds, where we set $T_{0}:=0$. For the sake of clarity, let us remark that in the current setting, the barriers in (18) and their parameters become

$$
\ell^{i j}(t):= \begin{cases}L_{i j} \mathrm{e}^{-(r-\kappa)\left(T_{i j}-t\right)}, & 0 \leq t<T_{i j} \\ M_{i j}, & t=T_{i j}\end{cases}
$$

From the issuer's point of view, the question of whether to postpone or not the face value $K$ payment depends on which alternative is cheaper. Hence, similarly to the situation of Bermuda options (see for instance [37]), the discounted price of a CoCo belonging to Additional Tier 1 capital category equals

$$
\widetilde{\Pi}_{t}:= \begin{cases}\inf _{\theta \in \mathscr{T}_{n}} \mathbb{E}^{*}\left[\widetilde{Z}_{\theta}^{(n)} \mid \mathscr{F}_{T_{n}}\right], & t=T_{n} \in\left\{T_{i}, \in \mathbb{N}\right\} \\ \mathbb{E}^{*}\left[\widetilde{\pi}_{T_{n+1}} \mid \mathscr{F}_{t}\right], & t \in\left(T_{n}, T_{n+1}\right),\end{cases}
$$

where $\mathscr{T}_{n}$ stands for the set of stopping times taking values in $\left\{T_{i}, i \geq n\right\}$ and

$$
\widetilde{Z}_{\theta}^{(n)}=\sum_{i=n+1}^{l: T_{l}=\theta} \sum_{j=1}^{m} \widetilde{c}_{i j} \mathbf{1}_{\left\{\tau_{i j}>T_{i j}, S_{T_{i j}}>M_{i j}\right\}}+K \mathrm{e}^{-r \theta} \mathbf{1}_{\{\tau>\theta\}}+\frac{K}{C_{p}} \mathrm{e}^{\kappa \tau} \widetilde{S}_{\tau} \mathbf{1}_{\{\tau \leq \theta\}} .
$$

It is important to remark that even though the general optimal stopping theory allows us to characterize the solution to the optimization problem in (48a), this is not enough to tackle whole pricing problem. Indeed, the solution to (48a) must be obtained in a relatively explicit way in order to be able to give a reasonable expression for the price in-between call dates (48b). Here we shall address the finite horizon case, i.e., the case where there are only finitely many call dates $\left\{T_{1}, \ldots, T_{N}\right\}$ and $T_{N}<\infty$.

In this case, for every fixed $T_{n}$, the solution to the optimization problem in (7.6.1) is related to the lower Snell envelope $\left(\widetilde{Y}_{k}^{(n)}\right)_{k \in\{n, \ldots, N\}}$ of the process $\left(\widetilde{X}_{k}^{(n)}\right)_{k \in\{n, \ldots, N\}}$ given by

$$
\widetilde{X}_{k}^{(n)}:=\widetilde{Z}_{T_{k}}^{(n)}=K \mathrm{e}^{-r T_{k}} \mathbf{1}_{\left\{\tau>T_{k}\right\}}+\frac{K}{C_{p}} \mathrm{e}^{\kappa \tau} \widetilde{S}_{\tau} \mathbf{1}_{\left\{\tau \leq T_{k}\right\}}+\sum_{i=n+1}^{k} \sum_{j=1}^{m} \widetilde{c}_{i j} \mathbf{1}_{\left\{\tau_{i j}>T_{i j}, S_{T_{i j}}>M_{i j}\right\}} .
$$

Having a finite horizon allows us to obtain $\left(\widetilde{Y}_{k}^{(n)}\right)_{k \in\{n, \ldots, N\}}$ by means of the following backwards procedure

$$
\widetilde{Y}_{k}^{(n)}= \begin{cases}\widetilde{X}_{N}^{(n)} & k=N \\ \min \left\{\widetilde{X}_{k}^{(n)}, \mathbb{E}^{*}\left[\widetilde{Y}_{k+1}^{(n)} \mid \mathscr{F}_{T_{k}}\right]\right\}, & k=N-1, \ldots, n .\end{cases}
$$


As it turns out, the lower Snell envelope $\left(\widetilde{Y}_{k}^{(n)}\right)_{k \in\{n, \ldots, N\}}$ can be obtained in a rather explicit form. Indeed, for the first iteration of (49), if $\tau>T_{n}$ then what we have is the raw expression

$$
\begin{aligned}
& \tilde{Y}_{N-1}^{(n)}=\min \left\{\widetilde{X}_{N-1}^{(n)}, \mathbb{E}^{*}\left[\tilde{X}_{N}^{(n)} \mid \mathscr{F}_{T_{N-1}}\right]\right\} \\
& =\min \left\{K \mathrm{e}^{-r T_{N-1}} \mathbf{1}_{\left\{\tau>T_{N-1}\right\}}+\frac{K}{C_{p}} \mathrm{e}^{\kappa \tau} \widetilde{S}_{\tau} \mathbf{1}_{\left\{\tau \leq T_{N-1}\right\}}+\sum_{i=n+1}^{N-1} \sum_{j=1}^{m} \widetilde{c}_{i j} \mathbf{1}_{\left\{\tau_{i j}>T_{i j},\right.}, S_{\left.T_{i j}>M_{i j}\right\}},\right. \\
& \left.\mathbb{E}^{*}\left[K \mathrm{e}^{-r T_{N}} \mathbf{1}_{\left\{\tau>T_{N}\right\}}+\frac{K}{C_{p}} \mathrm{e}^{\kappa \tau} \widetilde{S}_{\tau} \mathbf{1}_{\left\{\tau \leq T_{N}\right\}}+\sum_{i=n+1}^{N} \sum_{j=1}^{m} \widetilde{c}_{i j} \mathbf{1}_{\left\{\tau_{i j}>T_{i j},\right.}, S_{\left.T_{i j}>M_{i j}\right\}} \mid \mathscr{F}_{T_{N-1}}\right]\right\} \\
& =\frac{K}{C_{p}} \mathrm{e}^{\kappa \tau} \widetilde{S}_{\tau} \mathbf{1}_{\left\{\tau \leq T_{N-1}\right\}}+\sum_{i=n+1}^{N-1} \sum_{j=1}^{m} \widetilde{c}_{i j} \mathbf{1}_{\left\{\tau_{i j}>T_{i j}, S_{i j}>M_{i j}\right\}} \\
& +\mathbf{1}_{\left\{\tau>T_{N-1}\right\}} \min \left\{K \mathrm{e}^{-r T_{N-1}},\right. \\
& \left.\mathbb{E}^{*}\left[K \mathrm{e}^{-r T_{N}} \mathbf{1}_{\left\{\tau>T_{N}\right\}}+\frac{K}{C_{p}} \mathrm{e}^{\kappa \tau} \widetilde{S}_{\tau} \mathbf{1}_{\left\{\tau \leq T_{N}\right\}}+\sum_{j=1}^{m} \widetilde{c}_{N j} \mathbf{1}_{\left\{\tau_{N j}>T_{N j}, S_{T_{N j}}>M_{N j}\right\}} \mid \mathscr{F}_{T_{N-1}}\right]\right\} \\
& =\frac{K}{C_{p}} \mathrm{e}^{\kappa \tau} \widetilde{S}_{\tau} \mathbf{1}_{\left\{\tau \leq T_{N-1}\right\}}+\sum_{i=n+1}^{N-1} \sum_{j=1}^{m} \widetilde{c}_{i j} \mathbf{1}_{\left\{\tau_{i j}>T_{i j}, S_{T_{i j}}>M_{i j}\right\}}+\mathbf{1}_{\left\{\tau>T_{N-1}\right\}} \min \left\{K \mathrm{e}^{-r T_{N-1}}, \widetilde{\pi}_{T_{N-1}}\right\} \text {, }
\end{aligned}
$$

where as in the previous section, $\tilde{\pi}_{T_{N-1}}$ denotes the price of $\mathrm{Co}$ Co here with maturity $T_{N}$ and coupon structure $\left(c_{N j}, T_{N j}, \tau_{N j}\right)_{j=1}^{m}$. Due to the share price Markovianity, the price $\tilde{\pi}_{T_{N-1}}$ can be seen as function of $S_{T_{N-1}}$; we denote this function simply by $\widetilde{\pi}_{T_{N-1}}(x)$. Now, as discussed in Sect.3.1, the CoCo has a positive Delta, thus the function $\tilde{\pi}_{T_{N-1}}(x)$ is increasing and we can find a value $S_{N-1}^{*}$ such that

$$
S_{N-1}^{*}:=\inf \left\{x>0: \tilde{\pi}_{N}(x) \geq K \mathrm{e}^{-r T_{N-1}}\right\} .
$$

Hence we obtain

$$
\begin{aligned}
\widetilde{Y}_{N-1}^{(n)}= & \frac{K}{C_{p}} \mathrm{e}^{\kappa \tau} \widetilde{S}_{\tau} \mathbf{1}_{\left\{\tau \leq T_{N-1}\right\}}+\sum_{i=n+1}^{N-1} \sum_{j=1}^{m} \widetilde{c}_{i j} \mathbf{1}_{\left\{\tau_{i j}>T_{i j}, S_{T_{i j}}>M_{i j}\right\}} \\
& \left.+K \mathrm{e}^{-r T_{N-1}} \mathbf{1}_{\left\{\tau>T_{N-1}, S_{T_{N-1}} \geq S_{N-1}^{*}\right\}}+\widetilde{\pi}_{T_{N-1}} \mathbf{1}_{\left\{\tau>T_{N-1}, S_{T_{N-1}}<S_{N-1}^{*}\right\}}\right\} \\
= & K \mathrm{e}^{-r T_{N-1}} \mathbf{1}_{\left\{\tau>T_{N-1}, S_{T_{N-1}} \geq S_{N-1}^{*}\right\}}+\frac{K}{C_{p}} \mathrm{e}^{\kappa \tau} \widetilde{S}_{\tau} \mathbf{1}_{\left\{\tau \leq T_{N-1}\right\}}+\sum_{i=n+1}^{N-1} \sum_{j=1}^{m} \widetilde{c}_{i j} \mathbf{1}_{\left\{\tau_{i j}>T_{i j}, S_{i j}>M_{i j}\right\}} \\
& +\mathbb{E}^{*}\left[K \mathrm{e}^{-r T_{N}} \mathbf{1}_{\left\{\tau>T_{N}, S_{T_{N-1}}<S_{N-1}^{*}\right\}}+\frac{K}{C_{p}} \mathrm{e}^{\kappa \tau} \widetilde{S}_{\tau} \mathbf{1}_{\left\{S_{T_{N-1}}<S_{N-1}^{*}, T_{N-1}<\tau \leq T_{N}\right\}}\right. \\
& \left.\sum_{j=1}^{m} \widetilde{c}_{N j} \mathbf{1}_{\left\{\tau_{N j}>T_{N j}, S_{T_{N j}}>M_{N j}, S_{T_{N-1}}<S_{N-1}^{*}\right\}} \mid \widetilde{F}_{T_{N-1}}\right]
\end{aligned}
$$

So far we can see that basically all indicator functions appearing originally in (50), has been augmented in (51) by an additional condition on $S_{T_{N-1}}$ (i.e., $S_{T_{N-1}}<S_{N-1}^{*}$ 
or $\left.S_{T_{N-1}} \geq S_{N-1}^{*}\right)$. In [14] it has been shown that the computation of the Snell envelop $\left(\widetilde{Y}_{k}^{(n)}\right)_{k \in\{n, \ldots, N\}}$ can be carried out by means of the above backward procedure. In fact, it can be seen that the payoff of a CoCo having the callability feature can be written in terms of

$$
\begin{aligned}
& c_{i j} \mathbf{1}_{\left\{\tau_{i j}>T_{i j}, S_{T_{0}}<S_{0}^{*}, \ldots, S_{T_{i-1}}<S_{i-1}^{*}, S_{T_{i j}}>M_{i j}\right\}} \text { at times } T_{i j}, i \geq 1, \\
& K \mathbf{1}_{\left\{\tau>T_{i}, S_{T_{0}}<S_{0}^{*}, \ldots, S_{T_{i-1}}<S_{i-1}^{*}, S_{T_{i}}>S_{i}^{*}\right\}} \text { at } T_{i}, i \geq 1, \\
& \frac{K}{C_{p}} \mathrm{e}^{\kappa \tau} S_{\tau} \mathbf{1}_{\left\{\lceil\tau\rceil \leq T_{N}, S_{T_{0}}<S_{0}^{*}, \ldots, S_{\lceil\tau\rceil-1}<S_{\lceil\tau\rceil-1}^{*}\right\}} \text { at } \tau,
\end{aligned}
$$

where $\lceil\tau\rceil$ is the element of $\left\{T_{1}, \ldots, T_{N}\right\}$ such that $\lceil\tau\rceil-1<\tau \leq\lceil\tau\rceil$, and the additional variables $S_{1}^{*}, \ldots, S_{N-2}^{*}$ are defined by analogous reasoning to that behind the obtainment of $S_{N-1}^{*}$. Here $S_{0}^{*}:=\infty$ and $S_{N}^{*}:=0$ are set by convention.

Proposition 12 If the CoCo with extension risk is active and conversion has not occurred, then its discounted arbitrage-free price is given by

$$
\begin{aligned}
\widetilde{\Pi}_{t}= & \sum_{i, j: T_{i j}>t} \widetilde{c}_{i j} \mathbb{P}^{*}\left(\tau_{i j}>T_{i j}, S_{T_{0}}<S_{0}^{*}, \ldots, S_{T_{i-1}}<S_{i-1}^{*}, S_{T_{i j}}>M_{i j}, \mid \mathscr{F}_{t}\right) \\
& +\sum_{i: T_{i}>t} \widetilde{K} \mathbb{P}^{*}\left(\tau>T_{i}, S_{T_{0}}<S_{0}^{*}, \ldots, S_{T_{i-1}}<S_{i-1}^{*}, S_{T_{i}}>S_{i}^{*} \mid \mathscr{F}_{t}\right) \\
& +\sum_{i=1}^{N} \frac{K}{C_{p}} \mathrm{e}^{\kappa\left(T_{i}-t\right)} \widetilde{S}_{t} \mathbb{P}^{(S)}\left(\tau \leq T_{i}, S_{T_{0}}<S_{0}^{*}, \ldots, S_{T_{i-1}}<S_{i-1}^{*}, S_{T_{i}} \geq S_{i}^{*} \mid \mathscr{F}_{t}\right)
\end{aligned}
$$

Proof With the explicit description of the payoff corresponding to the CoCo with extension risk, the result is obtained as in Proposition 2, here taking into account the following identity

$$
\begin{aligned}
\{\lceil\tau\rceil & \left.\leq T_{N}, S_{T_{0}}<S_{0}^{*}, \ldots, S_{\lceil\tau\rceil-1}<S_{\lceil\tau\rceil-1}^{*}\right\} \\
= & \uplus_{i=1}^{N}\left\{\tau \leq T_{i}, S_{T_{0}}<S_{0}^{*}, \ldots, S_{T_{i-1}}<S_{i-1}^{*}, S_{T_{i}} \geq S_{i}^{*}\right\} .
\end{aligned}
$$

In view of this proposition, the obtainment of a closed-form formula for the price CoCo with extension risk requires the knowledge of the conditional distribution of $\left(\tau, S_{T_{0}}, S_{T_{1}}, \ldots, S_{T_{i}}\right)$ for $i=1, \ldots, N$. In the Black-Scholes model, this can be achieved by means of the following general lemma obtained in [14].

Lemma 13 Let $\left(B_{t}\right)_{t \geq 0}$ be a Brownian motion with drift $\mu$ and volatility $\sigma$, and denote by $\tau$ its first-passage time to level zero. Then, for arbitrary instants $T_{1}<$ $\cdots<T_{n}$ and arbitrary non-negative constants $a_{1}, \ldots, a_{n}$, on $\{\tau>t\}$ the following equation holds true 


$$
\begin{aligned}
\mathbb{P} & \left(\tau \geq T_{n}, B_{T_{1}}<a_{1}, \ldots, B_{T_{n-1}}<a_{n-1}, B_{T_{n}}>a_{n} \mid \mathscr{F}_{t}\right) \\
= & \mathbb{P}\left(-a_{1}<B_{T_{1}}<a_{1}, \ldots,-a_{n-1}<B_{T_{n-1}}<a_{n-1}, B_{T_{n}}>a_{n} \mid \mathscr{F}_{t}\right) \\
\quad & \quad \mathrm{e}^{-2 \mu \sigma^{-1} B_{t}} \mathbb{P}\left(-a_{1}<\bar{B}_{T_{1}}<a_{1}, \ldots,-a_{n-1}<\bar{B}_{T_{n-1}}<a_{n-1}, \bar{B}_{T_{n}}<-a_{n} \mid \mathscr{F}_{t}\right),
\end{aligned}
$$

where $\bar{B}_{T_{j}}=B_{T_{j}}-2 \mu\left(T_{j}-t\right), j=1, \ldots, n$, and $\left(\mathscr{F}_{t}\right)_{t \geq 0}$ stands for the natural filtration generated by $\left(B_{t}\right)_{t \geq 0}$.

Acknowledgments The work of J.M. Corcuera is supported by the Grant of the Spanish MCINN MTM2013-40782. This research was carried out at CAS -Centre for Advanced Study at the Norwegian Academy of Science and Letters, Research group SEFE.

Open Access This chapter is distributed under the terms of the Creative Commons Attribution Noncommercial License, which permits any noncommercial use, distribution, and reproduction in any medium, provided the original author(s) and source are credited.

\section{References}

1. Bertoin, J.: Lévy Processes. Cambridge University Press, Cambridge (1996)

2. Brigo, D., Garcia, J., Pede, N.: Coco bonds pricing with credit and equity calibrated firstpassage firm value models. Int. J. Theor. Appl. Financ. 18(3), 1550, 015 (2015). doi:10.1142/ S0219024915500156

3. Caballero, M., Pardo, J., Perez, J.: On the Lamperti stable processes. Probab. Math. Stat. 30, $1-28(2010)$

4. Carr, P., Geman, H., Madan, D., Yor, M.: The fine structure of asset returns: an empirical investigation. J. Bus. 75, 305-332 (2002)

5. Chan, S., van Wijnbergen, S.: CoCos, Contagion and Systemic Risk (14-110/VI/DSF79) (2014). http://ideas.repec.org/p/dgr/uvatin/20140110.html

6. Chen, N., Glasserman, P., Nouri, B., Pelger, M.: CoCos, bail-in, and tail risk. In: OFR Working paper. US Department of the Treasury (2013)

7. Cheridito, P., Xu, Z.: Pricing and hedging cocos. In: Social Science Research Network Working Paper Series (2013). http://dx.doi.org/10.2139/ssrn.2201364

8. Cheridito, P., Xu, Z.: A reduced form CoCo model with deterministic conversion intensity. In: Social Science Research Network Working Paper Series (2013). http://dx.doi.org/10.2139/ ssrn.2254403

9. Coculescu, D., Geman, H., Jeanblanc, M.: Valuation of default-sensitive claims under imperfect information. Financ. Stoch. 12, 195-218 (2008)

10. Collin-Dufresne, P., Goldstein, R., Helwege, J.: P. is credit event risk priced? modeling contagion via the updating of beliefs. In: Technical Report, National Bureau of Economic Research (2010)

11. Commission of Experts: Final report of the commission of experts for limiting the economic risks posed by large companies. In: Technical Report, Swiss National Bank (2010)

12. Corcuera, J.M., De Spiegeleer, J., Ferreiro-Castilla, A., Kyprianou, A.E., Madan, D.B., Schoutens, W.: Efficient pricing of contingent convertibles under smile conform models. J. Credit Risk 9(3), 121-140 (2013)

13. Corcuera, J.M., De Spiegeleer, J., Jönsson, H., Fajardo, J., Shoutens, W., Valdivia, A.: Close form pricing formulas for coupon cancellable cocos. J. Bank. Financ. (2014)

14. Corcuera, J.M., Fajardo, J., Schoutens, W., Valdivia, A.: CoCos with extension risk: a structural approach. In: Social Science Research Network Working Paper Series (2014). http://dx.doi. org/10.2139/ssrn.2540625 
15. Corcuera, J.M., Valdivia, A.: Short-term uncertainty. Working paper, University of Barcelona (2015)

16. De Spiegeleer, J., Schoutens, W.: Pricing contingent convertibles: a derivatives approach. J. Deriv. 20(2), 27-36 (2012)

17. De Spiegeleer, J., Schoutens, W.: Steering a bank around a death spiral: multiple trigger cocos. Wilmott 2012(59), 62-69 (2012)

18. De Spiegeleer, J., Schoutens, W.: CoCo bonds with extension risk. Wilmott 71, 78-91 (2014)

19. Dewatripont, M., Tirole, J.: The prudential regulation of banks. In: ULB-Universite Libre de Bruxelles, Technical Report (1994)

20. Dewatripont, M., Tirole, J.: Macroeconomic shocks and banking regulation. J. Money, Credit Bank. 44(s2), 237-254 (2012)

21. Duffie, D., Lando, D.: Term structure of credit spreads with incomplete accounting information. Econometrica 69, 633-664 (2001)

22. Flannery, M.: No pain, no gain? effecting market discipline via "reverse convertible debentures". In: Scott, H.S. (ed.) Capital Adequacy Beyond Basel: Banking, Securities, and Insurance, pp. 171-196 (2005)

23. Flannery, M.J.: Stabilizing large financial institutions with contingent capital certificates. In: CAREFIN Research Paper (04) (2010)

24. Gordy, M.B.: A risk-factor model foundation for ratings-based bank capital rules. J. Financ. Intermed. 12(3), 199-232 (2003)

25. Guo, X., Jarrow, A., Zeng, Y.: Credit risk models with incomplete information. Math. Oper. Res. 34(2), 320-332 (2009)

26. Heston, S.: A closed-form solution for options with stochastic volatility with applications to bond and currency options. Rev. Financ. Stud. 6(2), 327-343 (1993)

27. Jeanblanc, M., Valchev, S.: Partial information and hazard process. Int. J. Theor. Appl. Financ. 8, 807-838 (2005)

28. Koziol, C., Lawrenz, J.: Contingent convertibles. Solving or seeding the next banking crisis? J. Bank. Financ. 36(1), 90-104 (2012)

29. Kuznetsov, A., Kyprianou, A.E., Pardo, J.C.: A Wiener-Hopf Monte Carlo simulation technique for Lévy process. Ann. Appl. Probab. 21(6), 2171-2190 (2011)

30. Madan, D.B., Milne, F.: Option pricing with vg martingale components. Math. Financ. 1(4), 39-55 (1991)

31. Madan, D.B., Schoutens, W.: Break on through to the single side. J. Credit Risk 4(3), 3-20 (2008)

32. Pennacchi, G., Vermaelen, T., Wolff, C.: Contingent capital: the case for coercs. Available at SSRN 1785380 (2011)

33. Rogers, L.: Evaluating first-passage probabilities for spectrally one-sided Lévy processes. J. Appl. Probab. 37, 1173-1180 (2000)

34. Salminen, P., Borodin, A.: Handbook of Brownian Motion: Facts and Formulae. Birkhauser, Basel (1996)

35. Sato, K.: Lévy Processes and Infinitely Divisible Distributions. Cambridge Studies in Advanced Mathematics, vol. 68. Cambridge University Press, Cambridge (2000)

36. Schoutens, W., Cariboni, J.: Lévy Processes in Credit Risk. The Wiley Finance Series. Wiley, Chichester (2009)

37. Schweizer, M.: Advances in Finance and Stochastics. Essays in Honour of Dieter Sondermann., Chapter, On Bermudan Options, pp. 257-269. Springer (2002)

38. Stuart, A., Ord, J.: Kendall's Advanced Theory of Statistics. Distribution Theory, vol. 1. Wiley, Chichester (1994)

39. Sundaresan, S., Wang, Z.: On the design of contingent capital with a market trigger. J. Financ. 70(2), 881-920 (2015). doi:10.1111/jofi.12134 